\title{
Response Profiles to a Controlled Adverse Desiccating Environment Based on Clinical and Tear Molecule Changes.
}

Itziar Fernández, PhD;1,2 Alberto López-Miguel, PhD;2,3 Amalia Enríquez-deSalamanca, PhD;1,2 Marisa Tesón, PhD;² Michael E Stern, PhD;2,4 María J. González-García, PhD;1,2 Margarita Calonge, MD, PhD.1,2,4

Short title: Response profiles under adverse environment.

${ }^{1}$ Biomedical Research Networking Center in Bioengineering, Biomaterials and Nanomedicine (CIBER-BBN), Valladolid, Spain.

${ }^{2}$ IOBA (Institute of Applied Ophthalmobiology), University of Valladolid, Valladolid, Spain.

${ }^{3}$ Red Temática de Investigación Colaborativa en Oftalmología (OftaRed), Instituto de Salud Carlos III, Madrid, España.

${ }^{4}$ ImmunEyez LLC, CA, USA.

Corresponding author: Alberto López Miguel. IOBA, Universidad de Valladolid, Campus Universitario Miguel Delibes, Paseo de Belén 17, 47011, Valladolid, Spain. Telephone: +34983423274. Fax: +34983184723. Email: alopezm@ioba.med.uva.es

Conflict of interest: No conflicting relationship exists for any author. Disclosures of Dr. Margarita Calonge are the following: Research/clinical trials contracts, consultanships, advisory boards and/or lectures for Novaliq, Chiesi, Santen, Johnson and Johnson, Horus Pharma, Avizorex, and Roche laboratories. Disclosures of Dr. Michael Stern are the following: 
Research/clinical trials contracts, consultanships, advisory boards and/or lectures for Novaliq, Shire and Ocugen laboratories. The remaining authors have no relationship to disclose.

Funding: Supported in part by the Spanish Ministry of Science, Innovation and Universities, (Carlos III National Institute of Health) through Research Projects RETICS (RD16/008/0001 grant) (OftaRed); Biomedical Research Networking Center in Bioengineering; Biomaterials and Nanomedicine (CIBER-BBN); and SAF2016-77080-P grant from Agencia Estatal de Investigación (AEI) Ministry of Science, Innovation and Universities, Spain, and Fondo Europeo de Desarrollo Regional (FEDER), UE.

No funding organizations had a role in the design or conduct of this research. 


\section{ABSTRACT}

Purpose: To investigate response profiles in the lacrimal functional unit of dry eye disease (DED) and healthy volunteers after exposure to a controlled adverse desiccating environment (CADE) by identifying groups of individuals with similar clinical and molecular changes.

Methods: Clinical parameters and tear molecule levels of 20 mild-moderate DED patients and 20 healthy volunteers were evaluated pre- (baseline) and post-CADE exposure. Clustering based on relative change from baseline values was used to identify response profiles. One-vs-all logistic regression was used to identify baseline predictors for response clusters.

Results: Four response profiles were identified. Cluster 1: tear break-up time (TBUT) decrease and matrix metalloproteinase 9 (MMP-9) increase. Cluster 2: marked increase in corneal staining, up-regulation of both MMP-9 and interleukin (IL)-6 levels, and down-regulation of epithelial growth factor (EGF). Cluster 3: increase in fractalkine, vascular endothelial growth factor (VEGF), MMP-9, IL-6, IL-8, IL-1 receptor antagonist (IL-1Ra) and RANTES (regulated on activation, normal T expressed and secreted) tear levels; and increased corneal staining and decreased TBUT and phenol red thread scores. Cluster 4: decreased single-item score dry eye questionnaire (SIDEQ) scores and increased corneal staining. Predictive models using baseline variables found that cluster membership depended on: corneal and conjunctival staining, SIDEQ score, interferon gamma-induced protein (IP)-10, VEGF, and IL-1Ra concentrations. 
Conclusions: The response of both mild-moderate DED and healthy asymptomatic individuals to environmental stress (CADE) can be predicted based on baseline (pre-exposure) clinical and tear molecular parameters. Thus, identifying individuals with a predictable response could improve patient enrollment in DED clinical trials.

Keywords: Dry eye disease; controlled adverse desiccating environment; clustering; predictors; clinical signs; tear molecules.

Abbreviations: AUC = area under the receiver operation characteristic curve; $\mathbf{B I C}=$ bayesian Information criterion; $\mathbf{B C V A}=$ best corrected visual acuity; $\mathbf{C I}=$ confidence interval; CADE $=$ controlled adverse desiccating environment; $\mathbf{C C L}$ $=$ Chemokine $[\mathrm{C}-\mathrm{C}$ motif] ligand; $\mathrm{CXCL}=$ Chemokine $[\mathrm{C}-\mathrm{X}-\mathrm{C}$ motif] ligand; CX3CL = Chemokine [C-X3-C motif] ligand; DED = dry eye disease; $\mathbf{D E R P}=$ desiccating environment response prediction; EGF = Epidermal Growth Factor; FC = fold change; IFN-g = interferon - gamma; IL-1b= Interleukin-1b; IL-1RA = Interleukin-1 Receptor Antagonist; IL-2 = Interleukin-2; IL-6 = Interleukin-6; IL-8 = Interleukin-8; IL-10 = Interleukin-10 ; IL-12p70 = Interleukin-12p70; IL-17A = Interleukin-17A; IP-10 = interferon- gamma- Induced Protein-10; LFU = lacrimal functional unit; LOOCV $=$ leave-one-out-cross-validation; MMP-9 $=$ matrix metalloproteinase-9; OSDI $=$ ocular surface disease index; $\mathbf{P C}=$ principal component; PCA $=$ principal component analysis; RANTES $=$ Regulated on Activation, Normal $\mathrm{T}$ cell Expressed and Secreted; ROC $=$ receiver operation characteristic; SIDEQ = single-item score dry eye questionnaire. 


\section{1. INTRODUCTION}

2 The influence of environmental conditions on the lacrimal functional unit (LFU)

3 has been already proven and revised [1]. The LFU is exposed constantly to

4 adverse environmental conditions. These adverse conditions are triggering

5 factors for exacerbating dry eye disease (DED) [2], mainly because tear

6 evaporation is increased [3]. Even normal subjects are also negatively affected

7 by adverse climate-related conditions [4].

8 Desiccating environmental conditions can be reliably reproduced using 9 controlled environmental laboratories, or customized goggles in which case the exposure is restricted to the periocular area $[1,5]$. In recent years, many studies have demonstrated the usefulness of these facilities to measure the clinical effect of adverse environmental conditions [6-12]. Moreover, taking into account the inflammatory nature of DED, changes in concentrations of tear molecules commonly associated with DED have also been reported after undergoing desiccating stress conditions [6,8,9]. These include interleukin (IL-) 6, epidermal growth factor (EGF) and matrix metalloproteinase-9 (MMP-9).

Controlled environment laboratories enable the standardization of environmental conditions through continuous regulation of temperature, humidity and airflow, or even barometric pressure. Thus, they are recommended to be used when planning clinical studies and especially, clinical trials on DED [13]. On the one hand, the large influence of environmental conditions could be a major drawback to demonstrate the clinical efficacy of treatments involving the LFU, such as DED. The ability to control the environment allows patients to be equally exposed to the same conditions, thus 
minimizing the potential confounding effect of the environment when evaluating DED therapy outcomes in clinical trials. In fact, environmental chambers (or customized goggles) have been already used to evaluate the safety and efficacy of anti-inflammatory DED therapies in clinical trials [14-16]. On the other hand, recruiting patients with a particular reaction to adverse environmental conditions provides a good opportunity to reduce sample sizes, because individuals with more reproducible and homogeneous responses, could be enrolled. However, it is necessary to take into consideration the wide variability in the individual response to adverse environmental conditions. Some authors have used environmental chambers for deciding which particular individuals should be included in a DED clinical trial [17]. Their aim was to identify participants showing DED worsening to adverse environmental conditions. This procedure can improve patient recruitment by identifying participants with the ability to exacerbate signs and symptoms, however, the cost and time of recruitment during screening might be increased.

A simpler and especially, least-cost solution, would be to identify patients with similar response profiles to a controlled environment based solely on screening data (clinical and tear molecular variables). Therefore, in the present study, we have used a clustering procedure to identify the response profiles based on changes induced by a 2-hour controlled adverse desiccating environment (CADE) on several DED signs and symptoms, as well as on tear molecule concentrations. Once these profiles have been defined, predictive models for them have been built using pre-exposure data alone.

\section{METHODS}

\subsection{Participants and study design}


50 This prospective cross-sectional study adhered to the tenets of the Declaration

51 of Helsinki. Informed consent was obtained from the subjects after explanation

52 of the nature and possible consequences of the study. The University of

53 Valladolid Ethics Committee approved the study.

$54 \quad$ Forty participants were recruited. The sample was composed of 20 mild and moderate DED patients (Level 1 and 2 disease as classified by the first International Dry Eye Workshop (DEWS) dry eye severity grading scheme [18]), and 20 healthy volunteers with similar age and gender distribution. The inclusion criteria for DED patients were ocular surface disease index (OSDI) above 12 and corneal fluorescein staining grade 1 or 2 (Oxford scale). While for control asymptomatic volunteers, the inclusion criteria were an OSDI score $<12$ and corneal fluorescein staining $\leq 1$ (Oxford scale). Exclusion criteria for all participants were contact lens wear, ocular surgery during the last 6 months, any acute or chronic ocular disease other than DED and use of any topical medication other than artificial tears. DED patients could have history of topical anti-inflammatory therapies (i.e. steroids or cyclosporine), but not during the previous 3 months, and only artificial tears were allowed. Pregnancy or nursing was also an exclusion criterion. exposure within the controlled environment laboratory (CELab) previously described [9]. The environmental conditions selected were a temperature of $23^{\circ} \mathrm{C}, 5 \%$ relative humidity, and localized airflow (mean velocity: $0.43 \mathrm{~m} / \mathrm{s}$ ). These conditions are referred to as CADE (controlled adverse desiccating environment). Participants were watching a documentary on a conventional light-emitting diode television monitor during the exposure. 


\subsection{Clinical tests}

Objective and subjective ocular clinical examinations were performed. The objective measures were: (i) Tear osmolarity (TearLab Corporation, San Diego, California, USA); (ii) Phenol red thread test (Menicon Company Ltd, Nagoya, Japan) to evaluate tear production; (iii) Conjunctival hyperemia in bulbar nasal and temporal areas based on the Efron scale [19]; (iv) TBUT was performed after instillation of $5 \mu \mathrm{L}$ of $2 \%$ sodium fluorescein and calculated as the average value of 3 repetitions; (v) Corneal fluorescein staining using a cobalt-blue filter over the light source of the slit-lamp biomicroscope (SL-8Z; Topcon Corp, Tokyo, Japan) and a yellow Wratten no.12 filter (Eastman Kodak, Rochester, New York, USA), 2 minutes after instillation of $5 \mu \mathrm{L}$ of $2 \%$ sodium fluorescein. The Oxford and a modified Baylor scheme [9] dividing the cornea into central, superior, temporal, inferior, and nasal areas were used; (vi) Conjunctival staining was evaluated using lissamine green strips (GreenGlo; HUB Pharmaceuticals, LLC, Rancho Cucamonga, California, USA), and according to the Oxford scheme in nasal and temporal areas; and (vii) Schirmer I test without topical anesthesia.

The subjective dry-eye feeling was evaluated by the modified single-item score dry eye questionnaire (SIDEQ) using a visual analog scale [9]. SIDEQ items were considered individually and jointly through averaging.

\subsection{Tear inflammatory molecule analysis}

A glass capillary tube (Drummond Scientific, Broomall, PA, USA) was used to collect 2- $\mu \mathrm{L}$ of tear sample. The samples were diluted and frozen as described previously [20]. Two commercial immune bead-based assays were used to 
analyze 16 molecules in the tear samples using a Luminex IS-100 equipment (Luminex Corporation, Austin, Texas, USA). The concentrations of epidermal growth factor (EGF); vascular endothelial growth factor (VEGF); chemokine [CX3-C motif] ligand 1 (CX3CL1)/fractalkine; chemokine [C-X-C motif] ligand 8 (CXCL8)/IL-8; chemokine [C-X-C motif] ligand 10 (CXCL10)/interferon gammainduced protein 10 (IP-10); interferon (IFN)-gamma; interleukin (IL)-1b; interleukin-1 receptor antagonist (IL-1RA); IL-2; IL-6; IL-10; IL-12p70; IL-17A; chemokine [C-C motif] ligand 5 (CCL5)/regulated on activation, normal $\mathrm{T}$ cell expressed and secreted (RANTES), and tumor necrosis factor (TNF)-alpha were measured simultaneously with a 15-plex assay (HCYTO-60K 15XMilliplex; Millipore Iberica, Spain). Matrix metalloproteinase-9 (MMP-9) concentration was measured in a separate assay with a MMP-9 single-plex assay (HMMP2-55K Panel 2; Milliplex), which recognized the MMP-9 inactive zymogen and MMP-9 active forms. The samples were analyzed according to the manufacturer's protocol as previously described [20]. Molecule concentrations were analyzed as base-2 log-transformed variables. Cytokine levels below the limit of detection were imputed using the robust regression on order statistics (robust ROS) method introduced by Helsel and Cohn [21] and implemented in the NADA (Non-detects And Data Analysis) R package [22]. Limits of detection and detection rates are shown in table A1 (Appendix A).

\subsection{Data analysis}

Quantitative variables were expressed as mean \pm standard deviation (SD). Median and interquartile range (IQR) were used to summarize distributions of ordinal variables.

Two datasets were considered: 


\subsubsection{CADE Effect dataset}

Thirty-two clinical and molecular variables evaluated immediately before and after the 2-hour exposure to CADE were used to identify and describe response profiles. The CADE effect for each clinical parameter was computed as the relative change from pre-exposure baseline values. To take into account the minimum and maximum boundary values, the rate of change per individual was calculated as the relative difference between post- and pre-exposure values with respect to the maximum change over the considered times. The CADE effect for each tear molecule was quantified by log2 fold change (FC). Up and down-changes of the same magnitude in tear molecule expression have negative and positive symmetrical log2 values, respectively. One log2 FC (post/pre) means that the post-exposure value is twice as large as the preexposure one; two log2 FC means that the post-exposure value is 4 times as large as the pre-exposure one, and so on. Analogously, if the log2 FC value is 1 , the post-exposure value is half of the pre-exposure one, and so on.

\subsubsection{CADE Response Prediction dataset}

This group of variables was used to identify baseline variables that may have been contributing to membership in a particular response profile (Cluster). Clinical and molecular variables evaluated immediately before exposure to CADE were included in this group. Additionally, age, gender, and OSDI score before exposure were added to this dataset.

\subsubsection{Definition of response profiles to $C A D E$}

The starting point was the CADE Effect dataset. Firstly, a pre-processing step was performed using the caret (Classification And Regression Training) $R$ 
package [23]. All variables that showed no changes in at least $60 \%$ of our sample were ignored in the subsequent analysis.

A principal component analysis (PCA) was performed for reducing overlap and redundancy in the previously selected informative CADE Effect variables. PCA produces uncorrelated components, called principal components (PCs). These PCs are estimated as linear combinations of original variables and defined in such a way that the first PC accounts for as much of the variability in the data as possible. And each succeeding PC has the highest variance possible under the constraint that it is orthogonal to the preceding components. In this work, we kept the PCs necessary to explain at least $95 \%$ of the total variability in the data. Since skewness and the magnitude of the variables influence the PCA results, each of the features was centered, scaled and applied a Box and Cox transformation [24] to reduce skewness prior to the application of PCA.

The following stage of the analysis was the unsupervised classification of our study participants based on their joint clinical and tear molecular changes. A clustering procedure was performed using the PCs identified in the PCA. Trimmed k-means was applied to define the response profiles (Clusters) [25]. This procedure is a robust variant of k-means clustering method where a known fraction a of outliers is trimmed off, and the remaining observations are clustered into $\mathrm{k}$ groups. Its implementation is available in the tclust (robust trimmed clustering) $\mathrm{R}$ package [26] and parameters $\mathrm{k}$ (number of groups) and $\alpha$ (trimming proportion) should be fixed in advance. Classification trimmed likelihoods curves [27] were used to choice for $k$ and $\alpha$ parameters. 
The idea that a clustering algorithm should produce consistent results when applied to data sampled from the same source was used to evaluate the stability of our output partition. We used the algorithm proposed by Hennig [28]. Repeatedly, we generated 500 overlapping subsamples of $75 \%$ of the original sample and without replacement. Each subsample was clustered individually and the resulting partition was compared by Jaccard similarity coefficient [29] to our final clustering output for the overlapping shared set of points. We computed a stability value for each cluster as the average of subsamples Jaccard indexes. Clusters with a stability value less than 0.6 were considered unstable. Values between 0.6 and 0.75 indicated a pattern in the data. Clusters with stability values above 0.85 were considered highly stable [30]. To test the validity of the final clusters and facilitate their interpretation, a profile analysis was conducted, including a descriptive summary of all variables in the CADE Effect dataset. Statistically significant changes in clinical parameters greater than $25 \%$ were considered relevant changes. For tear molecule levels, this threshold was established at 2-fold (1 log2-FC).

\subsubsection{Prediction of response profiles to CADE}

One-vs-all logistic regression was used to quantify the association between the response profile and CADE Response Prediction variables. On a first stage, each CADE Response Prediction variable separately was used as independent variable in the four (one per cluster) simple logistic regression analyses. Variables associated with a cluster at the $10 \%$ significance level were identified as potential predictors of the corresponding response profile. Then, potential predictors were evaluated simultaneously to fit four multivariate logistic regression models, a multivariate classifier per cluster. The final panel of 
197 predictors of a particular response profile was defined as the optimal subset of

198 its potential predictors, optimizing the Bayesian Information Criterion (BIC) by exhaustive search. The leave-one-out-cross-validation (LOOCV) procedure was used to estimate the prediction accuracy of the fitted models, and the receiver operation characteristic (ROC) curve analysis was used to assess the discriminate ability. The final models were evaluated according to the area under the ROC curve (AUC). In addition, sensitivity and specificity were obtained by setting an optimal threshold using the pROC (display and analyze ROC curves) R package [31].

\section{RESULTS}

Forty participants, 20 DED (14 females and 6 males) and 20 healthy (14 females and 6 males), were evaluated before and after the 2-hour exposure to CADE. Their ages ranged from 39 to 76 years, the mean age of DED group was $64.6 \pm 8.1$ years, and the healthy group was $59.1 \pm 8.4$ years.

\subsection{Detection of response profiles to CADE}

213 Table 1 summarizes the clinical and molecular parameters before and after 2214 hours of exposure to CADE effect. Twenty-one informative variables of the initial 32 clinical and molecular parameters were condensed into a smaller set of

216 components by PCA (Table 1). From the 21 centered, scaled and skewness217 corrected variables, the PCA discovered 14 statistically-independent 218 dimensions (PCs), which together explained $95.8 \%$ of the total variation 219 (Appendix A. Table A2). 
Using the PCs and before clustering, classification trimmed likelihoods curves revealed an optimal number of four clusters and a trimming proportion of 0.025 (Appendix B. Figure B1). Applying trimmed k-means algorithm with these parameters, 39 participants were classified into 4 clusters, and one participant was trimmed out. Stable Jaccard coefficients were obtained for all clusters (Cluster 1: 0.83; Cluster 2: 0.71; Cluster 3: 0.78; Cluster 4: 0.69). Figures 1 and 2 show clinical and molecular profiles, respectively, for each of the 4 clusters found. Numerical description is shown in Table A3 (Appendix A). The key characteristics of each cluster are summarized as follows, and groups are named to resemble their dominant features.

Cluster 1: Mild response. Eighteen (45\%) participants (11 DED patients and 7 healthy individuals) were classified within this cluster. This group exhibited no major relevant changes in the clinical features. Only TBUT showed an average decrease above $25 \%(-27.1 \%$; 95\% Cl: $-37.6 \%,-16.2 \%)$. MMP-9 was the only tear molecule whose levels increased (log2-FC: $1.17 ; 95 \% \mathrm{Cl}: 0.41,2.02)$. DED patients and 8 healthy individuals) were classified within this cluster. Individuals in this cluster suffered an important increase in corneal staining after CADE. Of particular note was the change in inferior and nasal corneal staining, with an increase of $60 \%(95 \% \mathrm{Cl}: 40 \%, 80 \%)$ and $53.3 \%(95 \% \mathrm{Cl}: 40 \%, 69.2 \%)$, respectively. Additionally, MMP-9 increased its tear level more than four times after exposure (log2-FC: $2.41 ; 95 \% \mathrm{Cl}: 1.13,3.63)$. Another two additional molecules showed approximately two-fold change: EGF decreased (log2-FC: 1.21; 95\%Cl: -1.8, -0.64), while IL-6 increased (log2-FC: 1.28; 95\%Cl: 0.57 , 

and 1 healthy individual) were assigned to this cluster. This group was mainly characterized by a tear molecular response, as most of the studied cytokines showed a significant change (Appendix A. Table A3). Particularly large, about 8fold, were the increases of IL-1Ra (log2-FC: $2.85 ; 95 \% \mathrm{Cl}: 1.76,3.94)$ and fractalkine (log2-FC: 2.78; 95\% $\mathrm{Cl}: 2.29,3.14$ ). Tear molecules that increased more than 4-fold were: VEGF (log2-FC: 2.51 ; 95\%Cl: 1.8, 3.22), MMP-9 (log2FC: 2.42 ; 95\%Cl: $0.41,4.83$ ), and IL-8 (log2-FC: $2.28 ; 95 \% \mathrm{Cl}: 1.45,3.27)$. Finally, RANTES (log2-FC: 1.81; 95\%Cl: 0.98, 2.85), and IL-6 (log2-FC: 1.76; 95\% Cl: $0.59,2.75)$ increased more than twice. Only EGF and IP-10 showed non-relevant changes. Regarding significant clinical changes, there were an increase in inferior corneal staining $(55.6 \% ; 95 \% \mathrm{Cl}: 33.3 \% ; 77.8 \%)$ and in Schirmer test value $(44 \% ; 95 \% \mathrm{Cl}: 9.3 \%, 78.6 \%)$, and a decrease in TBUT ($34.4 \%$; $95 \% \mathrm{Cl}:-60.6 \%,-7.8 \%)$ and phenol red thread test $(-32.9 \%$; $95 \% \mathrm{Cl}$ : $41.8 \%,-22.7 \%)$.

Cluster 4: Symptomatic adaptation response. This cluster included 5 (12.5\%) participants (1 DED patient and 5 healthy individuals). Their clinical response profile was similar to that of cluster 2 in terms of increased corneal staining, however SIDEQ scores showed lower values after CADE exposure, especially the dryness item $(-95 \% ; 95 \% \mathrm{Cl}:-100 \%,-85 \%)$. Besides, although the clinical profile was similar to that of cluster 2, none of the tear molecules showed a relevant change.

A participant was trimmed out. The molecular profile of this individual was atypical presenting very important decreases in all studied cytokines (Appendix A. Table A3). 
The percentage of DED patients was higher in cluster 3 and cluster 1 , but none of the pairwise comparisons was statistically significant (multiple comparison adjusted $p>0.30)$.

\subsection{Prediction of response profiles to CADE}

Table 2 summarizes the variables into CADE response profiles dataset by response profile (cluster). Figure 3 shows the associations among each of the 4 response profiles (clusters) previously established and each separate CADE response profile.

After fitting for each cluster a multivariate logistic regression based on the best subset of potential predictors, the optimal models included 3 potential predictors for cluster 1 and cluster 2; and only one predictor for cluster 3 and cluster 4 (Appendix A. Table A4). Table 3 shows the final estimated odds ratio in every particular case. Membership in cluster 1 was predicted by low scores of corneal staining and conjunctival staining in nasal area, and high levels of IP10. Low baseline level of IP-10, high scores of corneal staining in temporal area and low SIDEQ score were identified as predictors of cluster 2 membership. Low levels of VEGF served as a predictor of response profile related to cluster 3 and high levels of IL-1Ra for cluster 4.

After carrying out the internal validation of final multivariate classifiers by the LOOCV procedure, the four models were characterized by high discrimination ability, showing AUC values statistically different from 0.5. Sensitivity and specificity values ranged from $68 \%$ to $100 \%$. In all cases, sensitivity values were higher than specificity except for cluster 2, where the specificity was slightly higher than sensitivity value (Table 4). 
Figure 4 shows a summary of all the phases of the statistical analysis carried out, and the most relevant outcomes obtained in each one of them.

\section{DISCUSSION}

297 The use of controlled environments has been recommended to evaluate the 298 effects of DED therapies and to study the underlying mechanisms of this 299 disease [5,13]. Selection of DED patients with positive, reproducible and homogeneous responses to controlled conditions could improve patient recruitment by decreasing the variability and required sample sizes in clinical trials. In the current study, we have focused primarily on identifying response profiles based on changes of different clinical and molecular variables after 2hour exposure to CADE in our facility (CELab). Then, for each particular profile, we have selected baseline parameters that enabled us to predict the most likely profile (Cluster) that each participant can be suited in. Thus, recruitment procedures in clinical trials where all patients should be evaluated before and after adverse condition exposure [17], could be even simplified.

We identified four clusters with high stability values. A slight DED exacerbation (Cluster 1: Mild-response cluster) was the most common type of response profile in our sample. It must be taken into account that participants were mild-moderate DED patients and similarly aged control volunteers. In this profile, only TBUT and MMP-9 showed a clinically relevant change (decrease and increase, respectively). Although we considered as clinically relevant a $25 \%$ change for clinical variables, and a 2-fold change for tear molecule concentrations, it must be also highlighted that inferior corneal staining increased $23 \%$ in this cluster. The mild exacerbation observed in this cluster does not seem to be specific because it was, to a greater or lesser degree, 
observed across all clusters identified. Therefore, we considered these changes as a common basic response of the LFU to an adverse environment, regardless of the presence of DED. It may be expected that exposure to a desiccating environment would provoke an increase in tear evaporation, resulting in tear hyperosmolarity that leads to altered cellular mechanisms [32]. Tear hyperosmolarity triggers MMP-9 release, thus initiating an inflammation process [33]. Furthermore, hyperosmolarity is negatively associated with TBUT [34], and in DED patients, this measure of tear film stability is inversely correlated with MMP-9 levels [33]. Consistently, we have observed that the increase of MMP-9 tear levels and the decrease of TBUT (as well as inferior corneal staining) are common responses in our sample population. Thus, it could be considered one of the basic effects resulting from a desiccating stress exposure.

The other three clusters were comprised of participants showing a more severe response to the desiccating environment. A common feature of these three groups is a clinically relevant increase in corneal fluorescein staining. This variable has been commonly used as primary endpoint to assess efficacy in many DED clinical trials, and in fact, it is one of the best ways for assessing ocular surface damage and dysfunction [35].

At the molecular level, in addition to the explained tear MMP-9 increase, a reduction in EGF and an increase in IL-6 were observed in cluster 2 (Corneal epithelial integrity response cluster). Change in the tear concentration of these 3 tear molecules have been widely reported in DED patients. A decreased concentration of EGF has already been associated with different types of DED patients $[36,37]$. Besides, IL-6 is a pro-inflammatory molecule frequently overexpressed in DED patients $[37,38]$. Moreover, this tear molecule rapidly 
344 increases when subjecting in vitro corneal epithelial cells to a short term desiccation (30 minutes) [39]. From a clinical viewpoint, this cluster is mainly characterized by a great increase of corneal fluorescein staining.

Individuals within cluster 3 (Tear molecular response cluster) were mainly characterized by a great up-regulation of pro-inflammatory tear molecules. It was observed a great acute inflammatory response involving modifications in concentrations of all tear molecules evaluated. These individuals clearly showed a great imbalance in the LFU, which overreacted to the corneal insult secreting a huge amount of cytokines and chemokines as well as MMP-9.

Finally, in cluster 4 (Symptomatic adaptation response cluster), individuals were mainly characterized by their symptomatic response to the desiccating exposure. They also showed an increase in corneal staining, and, in contrast to those of the other clusters, these participants reported a marked recovery in dry eye symptoms. This phenomenon has been previously published by Ousler et al [40]. These authors demonstrated that healthy and mild-moderate DED patients exposed to adverse conditions can show a worsening in ocular discomfort followed by a temporary improvement, in contrast to severe DED patients who do not follow this pattern. This scenario was explained as a natural compensation to the adverse environment using mechanisms like blinking and tearing. Besides, it is well known that there is a poor correlation between symptoms and DED signs [41]. Thus, if DED-related symptoms are to be selected as primary end-point in a clinical trial, cluster 4 individuals should not be recruited as they are not likely to report differences in symptoms between experimental and control medications. 
Using cluster analysis, we were able to find four different patterns of response to a desiccating environment (CADE) based on changes in clinical and tear molecular variables. The cluster analysis has demonstrated that there are different types of responses to the same environmental stimuli depending in each individual. Although the identification and interpretation of these response profiles might be restricted to our sample population, it would be genuinely useful to be able to classify individuals into response subgroups before undergoing desiccating stress when performing clinical studies and trials. This methodology would reduce recruitment time and clinical trial costs. The predictors that we found were not only clinical (corneal and conjunctival staining as well as modified SIDEQ score) variables, but also biochemical ones (IL-1Ra, IP-10 and VEGF tear levels). This finding shows that, in addition to clinical ocular examination, it is worth assessing tear molecular status as well in DED patients recruited for clinical trials [42]. Taking into account that pivotal phase III trials are necessary to get marketing approvals from regulatory agencies worldwide, it could be interesting to perform cluster analysis and fitting classifiers (clinical and biochemical) during phase II trials. This methodology could improve patient recruitment and selection of efficacy end-points for phase III clinical trials. simplicity. However, when dimensionality increases, this algorithm could not work well. To improve its efficiency, we applied PCA on original data set and obtained a reduced dataset containing uncorrelated variables. Hence, clustering was performed in a lower-dimensional dataset and the resulting clusters may be 
more meaningful. On the other hand, the K-means result may not be accurate due to presence of outliers, participants that are different from (or inconsistent with) the rest of the recruited individuals. Moreover, the influence of outliers will be more important when the sample size is small, since typically larger sample sizes allow more accurate estimations. To overcome this problem, trimmed kmeans was performed [25]. In regard to the prediction step, some authors recommended a minimum sample of 10 events per independent variable in a logistic regression [43], although more recent simulation analysis suggested that this rule can be too conservative [44]. A first attempt to reduce the number of predictors was to select as a candidate for the multivariate analysis only those variables having a significant univariate test at the $10 \%$ level. This approach greatly reduced the problem, especially in the smaller cluster (Cluster $4, n=5)$. It is important to emphasize that we have found possible existing patterns and predictors that need to be validated in external samples. Nevertheless, these preliminary results look promising. Our evaluations, based on internal validation measures, were appropriate for both clustering and predicting stages. Additionally, the response profiles have shown a consistent interpretation with clinically meaningful outcomes. Another limitation is that the definition and identification of response patterns was carried out on data from a prospective study involving mild-to-moderate DED patients and asymptomatic participants. Therefore, any conclusion about these response profiles may not be appropriate for patients with severe epithelial damage and for also patients with no corneal staining but mild-moderate conjunctival damage and patients with no epithelial damage but decreased BUT and/or increased tear film 
417 osmolarity. Other dissimilar populations may have a slightly different response 418 to the desiccating stress exposure.

\section{5. CONCLUSIONS}

420 In conclusion, we showed that the response of most common DED patients 421 and control individuals to desiccating stress can be grouped into diverse 422 clusters. The response is always a deterioration of the LFU, however, 423 depending on each individual the response might be characterized differently. In 424 addition, we demonstrated that it might be possible to determine some clinical 425 and tear biochemical classifiers that could predict the response of each 426 individual (type of cluster) to desiccating stress. The ability to predict LFU 427 response is especially important, because it could be very useful to improve 428 recruitment in clinical trials that try to show therapeutic effectiveness in DED. 


\section{Disclosure/Conflict of Interest Statement}

No conflicting relationship exists for any author. Disclosures of Dr. Margarita Calonge are the following: Research/clinical trials contracts, consultanships, advisory boards and/or lectures for Novaliq, Chiesi, Santen, Johnson and Johnson, Horus Pharma, Avizorex, and Roche laboratories. Disclosures of Dr. Michael Stern are the following: Research/clinical trials contracts, consultanships, advisory boards and/or lectures for Novaliq, Shire and Ocugen laboratories. The remaining authors have no relationship to disclose. 


\section{REFERENCES}

1. Calonge M, Pinto-Fraga J, González-García MJ, Enríquez-de-Salamanca A, López-de la Rosa A, Fernández I, et al. Effects of the external environment on dry eye disease. Int Ophthalmol Cli 2017;57:23-40.

2. van Setten G, Labetoulle M, Baudouin C, Rolando M. Evidence of seasonality and effects of psychrometry in dry eye disease. Acta Ophthalmol 2016;94:499-506.

3. Bron AJ, Tomlinson A, Foulks GN, Pepose JS, Baudouin C, Geerling G, et al. Rethinking dry eye disease: a perspective on clinical implications. Ocul Surf 2014;12:S1-31.

4. Tesón M, López-Miguel A, Neves H, Calonge M, González-García MJ, González-Méijome JM. Influence of climate on clinical diagnostic dry eye tests: pilot study. Optom Vis Sci 2015;92:284-9.

5. Calonge M, Labetoulle M, Messmer EM, Shah S, Akova YA, Boboridis KG, et al. Controlled adverse environment chambers in dry eye research. Curr Eye Res 2018;43:445-50.

6. López-Miguel A, Tesón M, Martín-Montañez V, Enríquez-de-Salamanca A, Stern $\mathrm{ME}$, Calonge $\mathrm{M}$ et al. Dry eye exacerbation in patients exposed to desiccating stress under controlled environmental conditions. Am J Ophthalmol 2014;157:788-98.

7. Alex A, Edwards A, Hays JD, Kerkstra M, Shih A, de Paiva CS, et al. Factors predicting the ocular surface response to desiccating environmental stress. Invest Ophthalmol Vis Sci 2013;54:3325-32.

8. López-Miguel A, Tesón M, Martín-Montañez V, Enríquez-de-Salamanca A, Stern ME, González-García MJ, et al. Clinical and molecular inflammatory 
response in Sjögren syndrome-associated dry eye patients under desiccating stress. Am J Ophthalmol 2016;161:133-41.

9. Tesón M, González-García MJ, López-Miguel A, Enríquez-de-Salamanca A, Martín-Montañez V, Benito MJ, et al. Influence of a controlled environment simulating an in-flight airplane cabin on dry eye disease. Invest Ophthalmol Vis Sci 2013;54:2093-9.

10. Madden LC, Tomlinson A, Simmons PA. Effect of humidity variations in a controlled environment chamber on tear evaporation after dry eye therapy. Eye Contact Lens. 2013;39:169-74.

11. López-de la Rosa A, Martín-Montañez V, López-Miguel A, Fernández I, Calonge M, González-Méijome JM, et al. Ocular response to environmental variations in contact lens wearers. Ophthalmic Physiol Opt 2017;37:60-70.

12. Maruyama K, Yokoi N, Takamata A, Kinoshita S. Effect of environmental conditions on tear dynamics in soft contact lens wearers. Invest Ophthalmol Vis Sci 2004;45:2563-8.

13. Ousler GW, Gomes PJ, Welch D, Abelson MB. Methodologies for the study of ocular surface disease. Ocul Surf 2005;3:143-54.

14.Pinto-Fraga J, López-Miguel A, González-García MJ, Fernández I, Lópezde-la-Rosa A, Enríquez-de-Salamanca A, et al. Topical fluorometholone protects the ocular surface of dry eye patients from desiccating stress: A randomized controlled clinical trial. Ophthalmology 2016;123:141-53.

15. Tauber J, Karpecki P, Latkany R, Luchs J, Martel J, Sall K, et al. Lifitegrast ophthalmic solution $5.0 \%$ versus placebo for treatment of dry eye disease: results of the randomized phase III OPUS-2 study. Ophthalmology 2015;122:2423-31. 
16. Moore QL, De Paiva CS, Pflugfelder SC. Effects of dry eye therapies on environmentally induced ocular surface disease. Am J Ophthalmol 2015;160:135-42.

17. Semba CP, Torkildsen GL, Lonsdale JD, McLaurin EB, Geffin JA, Mundorf TK, et al. A phase 2 randomized, double-masked, placebo-controlled study of a novel integrin antagonist (SAR 1118) for the treatment of dry eye. Am J Ophthalmol 2012;153:1050-60.

18. Lemp AM, Baudouin C, Baum J, Dogru M, Foulks GN, Kinoshita S, et al. The definition and classification of dry eye disease: report of the Definition and Classification Subcommittee of the International Dry Eye WorkShop (2007). Ocul Surf 2007;5:75-92.

19. Efron N. Grading scales for contact lens complications. Ophthalmic Physiol Opt 1998;18:182-6.

20. Enriquez-de-Salamanca A, Castellanos E, Stern ME, Fernández I, Carreño E, García-Vázquez C, et al. Tear cytokine and chemokine analysis and clinical correlations in evaporative-type dry eye disease. Mol Vis 2010;16:862-73.

21. Helsel DR, Cohn TA. Estimation of descriptive statistics for multiply censored wáter quality data. Water Resour Re. 1988;24:1997-2004.

22. Lee L. NADA: Nondetects and Data Analysis for Environmental Data. $R$ package version 1.6-1. https://CRAN.R-project.org/package=NADA; 2017 Accessed April 2017.

23. Kuhn M. caret: Classification and regression training. $R$ package version 6.0-76. https://CRAN.R-project.org/package=caret; 2017 Accessed April 2017. 
24.Box G, Cox D. An analysis of transformations. J R Stat Soc Series B Stat Methodol 1964;211-52.

25. Cuesta-Albertos JA, Gordaliza A, Matrán C. Trimmed k-means: an attempt to robustify quantizers. Ann Stat 1997;25:553-76.

26. Fritz H, Garcia-Escudero LA, Mayo-Iscar A. tclust: An R Package for a trimming approach to cluster analysis. J Stat Softw 2012;47:1-26.

27. Garcia-Escudero LA, Gordaliza A, Matrán C, Mayo-Iscar A. Exploring the number of groups in robust model-based clustering. Stat Comput 2011;21:585-99.

28. Hennig C. Cluster-wise assessment of cluster stability. Comput Stat Data Anal 2007;52:258-71.

29. Jaccard S. Nouvelles recherches sur la distribution florale. Bull Soc Vaud Sci Nat 1908;44:223-70.

30. Hennig C. Dissolution point and isolation robustness: robustness criteria for general cluster analysis methods. J Multivar Anal 2008;99:1154-76.

31. Robin X, Turck N, Hainard A, Tiberti N, Lisacek F, Sanchez JC, et al. pROC: an open-source package for R and S+ to analyze and compare ROC curves. BMC Bioinformatics 2011;12:77.

32. Mantelli F, Massaro-Giordano M, Macchi I, Lambiase A, Bonini S. The cellular mechanisms of dry eye: from pathogenesis to treatment. J Cell Physiol 2013;228:2253-6.

33. Aragona P, Aguennouz M, Rania L, Postorino E, Sommario MS, Roszkowska AM, et al. Matrix metalloproteinase 9 and transglutaminase 2 expression at the ocular surface in patients with different forms of dry eye disease. Ophthalmology 2015;122:62-71. 
34. Masmali A, Alrabiah S, Alharbi A, El-Hiti GA, Almubrad T. Investigation of tear osmolarity using TearLab Osmolarity System in normal adults in Saudi Arabia. Eye Contact Lens 2014;40:74-8.

35. Tsubota K, Ashell P, Dogru M, Fonn D, Foulks G, Schaumberg D, et al. Design and conduct of clinical trials: report of the clinical trials subcommittee of the international dry eye workshop (2007). Ocul Surf 2007;5:153-62.

36. Cocho L, Fernández I, Calonge M, Martínez V, González-García MJ, Caballero D, et al. Biomarkers in ocular chronic graft versus host disease: tear cytokine- and chemokine-based predictive model. Invest Ophthalmol Vis Sci 2016;57:746-58.

37. Lam H, Bleiden L, De Paiva CS, Farley W, Stern ME, Pflugfelder SC. Tear cytokine profiles in dysfunctional tear syndrome. Am J Ophthalmol 2009;147:198-205.

38. Na KS, Mok JW, Kim JY, Rho CR, Joo CK. Correlations between tear cytokines, chemokines, and soluble receptors and clinical severity of dry eye disease. Invest Ophthalmol Vis Sci 2012;53:5443-50.

39. Higuchi A, Kawakita T, Tsubota K. IL-6 induction in desiccated corneal epithelium in vitro and in vivo. Mol Vis 2011;17:2400-6.

40. Ousler GW 3rd, Abelson MB, Nally LA, Welch D, Casavant JS. Evaluation of the time to "natural compensation" in normal and dry eye subject populations during exposure to a controlled adverse environment. Adv Exp Med Biol 2002;506:1057-63.

41. Vehof J, Sillevis Smitt-Kamminga N, Nibourg SA, Hammond CJ. Predictors of discordance between symptoms and signs in dry eye disease. Ophthalmology 2017;124:280-6. 
42. Pinto-Fraga J, Enríquez-de-Salamanca A, Calonge M, González-García MJ, López-Miguel A, López-de la Rosa A, et al. Severity, therapeutic, and activity tear biomarkers in dry eye disease: An analysis from a phase III clinical trial. Ocul Surf 2018;16:368-76.

43. Peduzzi P, Concato J, Kemper E, Holford TR, Feinstein AR. A simulation study of the number of events per variable in logistic regression analysis. $\mathrm{J}$ Clin Epidemiol 1996;49:1373-9.

44. Vittinghoff E, McCulloch CE. Relaxing the rule of ten events per variable in logistic and cox regression. Am J Epidemiol 2007;165:710-8. 


\section{$\underline{\text { TABLES }}$}

Table 1. Clinical data and tear molecule levels before and 2 hours after exposure to a controlled adverse desiccating environment (CADE). CADE effect for each clinical parameter was computed as the relative change (percentage) from pre-exposure time. For each tear molecule level this effect was quantified by log2-Fold change (FC). Non change percentage (last column) is the percentage of participants not having a modification in the parameter score after 2 hours of CADE. For clinical and molecular parameters $0 \%$ and 0 log2-FC are the no-change values, respectively. Variables showing no changes in at least $60 \%$ of sample were considered as non-informative features.

\begin{tabular}{|c|c|c|c|c|c|}
\hline \multirow[b]{2}{*}{ Parameters } & \multirow{2}{*}{$\begin{array}{l}\text { Before CADE } \\
\text { Mean } \pm \text { SD or } \\
\text { Median } \pm I Q R\end{array}$} & \multirow{2}{*}{$\begin{array}{c}2 \text { hour after CADE } \\
\text { Mean } \pm \text { SD or } \\
\text { Median } \pm I Q R \\
\end{array}$} & \multicolumn{3}{|c|}{ CADE effect } \\
\hline & & & Mean \pm SD & $\begin{array}{c}\text { Non change } \\
\text { percentage }(95 \% \mathrm{CI})\end{array}$ & $\begin{array}{c}\text { Informative } \\
\text { variable } \\
\end{array}$ \\
\hline \multicolumn{6}{|l|}{ Conjunctival hyperemia } \\
\hline Nasal & $1 \pm 1$ & $1 \pm 1$ & $5.8 \pm 16.1$ & $75 \%(58.5 \% ; 86.8 \%)$ & \\
\hline Temporal & $1 \pm 0$ & $1 \pm 1$ & $4.6 \pm 21.6$ & $67.5 \%(50.8 \% ; 80.9 \%)$ & \\
\hline Tear osmolarity $(\mathrm{mOsm} / \mathrm{l})$ & $317.2 \pm 22.9$ & $318.2 \pm 22$ & $0.5 \pm 6.7$ & $2.5 \%(0.1 \% ; 14.7 \%)$ & $\checkmark$ \\
\hline Phenol red thread $\mathrm{t}$ & $20.3 \pm 7.4$ & $19.4 \pm 6.9$ & $11.3 \pm 79.3$ & $0 \%(0 \% ; 10.9 \%)$ & $\checkmark$ \\
\hline TBUT & $2.8 \pm 1.8$ & $2.0 \pm 1.0$ & $-22.1 \pm 30.7$ & $22.5 \%(11.4 \% ; 38.9 \%)$ & $\checkmark$ \\
\hline Corneal staining (Oxford) & $0 \pm 1$ & $1 \pm 1$ & $13.9 \pm 13$ & $42.5 \%(27.4 \% ; 59 \%)$ & $\checkmark$ \\
\hline \multicolumn{6}{|l|}{ Corneal staining (Baylor) } \\
\hline Central & $0 \pm 0$ & $0 \pm 1$ & $6 \pm 23.4$ & $72.5 \%(55.9 \% ; 84.9 \%)$ & \\
\hline Nasal & $0 \pm 1$ & $1 \pm 2$ & $19.4 \pm 43.5$ & $32.5 \%(19.1 \% ; 49.2 \%)$ & $\checkmark$ \\
\hline Temporal & $0.5 \pm 1$ & $1 \pm 1.2$ & $19 \pm 29.9$ & $42.5 \%(27.4 \% ; 59 \%)$ & $\checkmark$ \\
\hline Superior & $0 \pm 0$ & $0 \pm 0$ & $5.6 \pm 15.5$ & $85 \%(69.5 \% ; 93.8 \%)$ & \\
\hline Inferior & $1 \pm 1$ & $2 \pm 1$ & $42.1 \pm 40.4$ & $17.5 \%(7.9 \% ; 33.4 \%)$ & $\checkmark$ \\
\hline Total & $2.4 \pm 2.3$ & $6 \pm 3.9$ & $20.1 \pm 18.4$ & $10 \%(3.3 \% ; 24.6 \%)$ & $\checkmark$ \\
\hline \multicolumn{6}{|l|}{ Conjunctival staining } \\
\hline Nasal & $1 \pm 1$ & $1 \pm 1$ & $5.2 \pm 10$ & $77.5 \%(61.1 \% ; 88.6 \%)$ & \\
\hline Temporal & $0.5 \pm 1$ & $0.5 \pm 1$ & $-2.8 \pm 32.1$ & $70 \%(53.3 \% ; 82.9 \%)$ & \\
\hline Schirmer test & $12.2 \pm 9.3$ & $14.1 \pm 10.7$ & $34.4 \pm 77.1$ & $17.5 \%(7.9 \% ; 33.4 \%)$ & $\checkmark$ \\
\hline \multicolumn{6}{|l|}{ SIDEQ } \\
\hline Dry eye & $1.4 \pm 1.9$ & $1.5 \pm 2$ & $-11.8 \pm 40.2$ & $52.5 \%(36.3 \% ; 68.2 \%)$ & $\checkmark$ \\
\hline Foreign body sensations & $1.6 \pm 2.4$ & $1.4 \pm 2$ & $-9.4 \pm 29.5$ & $47.5 \%(31.8 \% ; 63.7 \%)$ & $\checkmark$ \\
\hline Burning & $1.2 \pm 2$ & $1.1 \pm 1.7$ & $-3.5 \pm 18.7$ & $62.5 \%(45.8 \% ; 76.8 \%)$ & \\
\hline Pain & $0.6 \pm 1.4$ & $0.4 \pm 1.2$ & $-7.9 \pm 27.6$ & $77.5 \%(61.1 \% ; 88.6 \%)$ & \\
\hline Itching & $0.9 \pm 2$ & $1 \pm 1.9$ & $-0.1 \pm 10.6$ & $73.7 \%(56.6 \% ; 86 \%)$ & \\
\hline Photophobia & $1.1 \pm 2.3$ & $0.9 \pm 1.7$ & $-12.6 \pm 38.3$ & $60.5 \%(43.5 \% ; 75.5 \%)$ & \\
\hline Blurred vision & $0.7 \pm 1.7$ & $0.7 \pm 1.6$ & $-8.4 \pm 34.4$ & $69.2 \%(52.3 \% ; 82.5 \%)$ & \\
\hline Average & $1.1 \pm 1.6$ & $1 \pm 1.5$ & $-13.2 \pm 30.3$ & $35 \%(21.1 \% ; 51.7 \%)$ & $\checkmark$ \\
\hline
\end{tabular}




\begin{tabular}{|c|c|c|c|c|c|}
\hline & Before CADE & 2 hour after CADE & \multicolumn{3}{|c|}{ CADE effect } \\
\hline Parameters & $\begin{array}{l}\text { Mean } \pm \text { SD or } \\
\text { Median } \pm I Q R\end{array}$ & $\begin{array}{l}\text { Mean } \pm \text { SD or } \\
\text { Median } \pm I Q R\end{array}$ & Mean \pm SD & $\begin{array}{c}\text { Non change } \\
\text { percentage }(95 \% \mathrm{CI})\end{array}$ & $\begin{array}{c}\text { Informative } \\
\text { variable } \\
\end{array}$ \\
\hline \multicolumn{6}{|c|}{ Tear molecule levels $(\mathrm{pg} / \mathrm{mL})$ detected in at least $80 \%$ of participants } \\
\hline EGF & $1683.6 \pm 1431.3$ & $991.4 \pm 731.2$ & $-64.1 \pm 139.7$ & $0 \%(0 \% ; 10.9 \%)$ & $\checkmark$ \\
\hline CX3CL1/ Fractalkine & $1068.3 \pm 990$ & $1016.8 \pm 1075.5$ & $16.2 \pm 176.1$ & $2.5 \%(0.1 \% ; 14.7 \%)$ & $\checkmark$ \\
\hline IL-1Ra & $7488.4 \pm 7198.5$ & $7588.7 \pm 8371.1$ & $16.5 \pm 239.4$ & $0 \%(0 \% ; 10.9 \%)$ & $\checkmark$ \\
\hline IL-6 & $56.8 \pm 104.3$ & $61 \pm 45.7$ & $75.7 \pm 137.6$ & $2.5 \%(0.1 \% ; 14.7 \%)$ & $\checkmark$ \\
\hline CXCL8/ IL-8 & $859.1 \pm 1350.8$ & $856.6 \pm 777.6$ & $36.7 \pm 140.2$ & $0 \%(0 \% ; 10.9 \%)$ & $\checkmark$ \\
\hline CXCL10/ IP-10 & $54692.8 \pm 66230.5$ & $57969.8 \pm 64277.3$ & $13.3 \pm 181.7$ & $0 \%(0 \% ; 10.9 \%)$ & $\checkmark$ \\
\hline CCL5/ RANTES & $20.9 \pm 14.6$ & $42.1 \pm 109.2$ & $37 \pm 141.5$ & $2.5 \%(0.1 \% ; 14.7 \%)$ & $\checkmark$ \\
\hline VEGF & $641 \pm 677.9$ & $636.9 \pm 475.8$ & $41.4 \pm 144.9$ & $2.5 \%(0.1 \% ; 14.7 \%)$ & $\checkmark$ \\
\hline MMP-9 & $12006.3 \pm 36722.2$ & $20861.9 \pm 59663.6$ & $162.9 \pm 210.4$ & $0 \%(0 \% ; 10.9 \%)$ & $\checkmark$ \\
\hline
\end{tabular}

$\mathrm{SD}=$ Standard Deviation; $\mathrm{IQR}=$ Interquartile Range; $\mathrm{CI}=$ Confidence interval; TBUT $=$ Tear film Break-Up Time; SIDEQ = Single-Item Score Dry Eye Questionnaire; EGF = Epidermal Growth Factor; $\mathrm{CX} 3 \mathrm{CL}=$ Chemokine $[\mathrm{C}-\mathrm{X} 3-\mathrm{C}$ motif] ligand; IL-1RA = Interleukin-1 Receptor Antagonist; IL-6 = Interleukin-6; CXCL = Chemokine [C-X-C motif] ligand; IL-8 = Interleukin-8; IP-10 = interferon- $\gamma$ - Induced Protein-10; CCL $=$ Chemokine [C-C motif] ligand; RANTES $=$ Regulated on Activation, Normal $\mathrm{T}$ cell Expressed and Secreted; VEGF $=$ Vascular Endothelial Growth Factor; MMP-9 = matrix metalloproteinase-9. 
Table 2. Description of controlled adverse desiccating environment (CADE) response prediction dataset for each cluster (response profile). Mean and standard deviation was used to describe quantitative variables. For ordinal variables, median and interquartile range are shown in italic font. For gender, the percentage of males (and its $95 \%$ confidence interval) is calculated.

\begin{tabular}{|c|c|c|c|c|}
\hline & $\begin{array}{c}\text { Cluster } 1(\mathbf{n}=\mathbf{1 8}) \\
\text { Mild response }\end{array}$ & $\begin{array}{l}\text { Cluster } \mathbf{2}(\mathbf{n}=\mathbf{1 0}) \\
\text { Corneal epithelial } \\
\text { integrity response }\end{array}$ & $\begin{array}{l}\text { Cluster } \mathbf{3}(\mathbf{n}=\mathbf{6}) \\
\text { Tear molecular } \\
\text { response }\end{array}$ & $\begin{array}{c}\text { Cluster } \mathbf{4}(\mathbf{n}=\mathbf{5}) \\
\text { Symptomatic } \\
\text { adaptation response }\end{array}$ \\
\hline \multicolumn{5}{|l|}{ Demographic parameters } \\
\hline Age & $61.9 \pm 9.6$ & $60.2 \pm 7.7$ & $66.8 \pm 4.6$ & $57.8 \pm 10.3$ \\
\hline Gender (male) & $44.4 \%(22.4 \% ; 68.7 \%)$ & $20 \%(3.5 \% ; 55.8 \%)$ & $16.7 \%(0.9 \% ; 63.5 \%)$ & $20 \%(1.1 \% ; 70.1 \%)$ \\
\hline \multicolumn{5}{|l|}{ Clinical parameters } \\
\hline $\begin{array}{l}\text { Conjunctival hyperemia } \\
\text { Nasal } \\
\text { Temporal }\end{array}$ & $\begin{array}{l}1 \pm 1 \\
1 \pm 0\end{array}$ & $\begin{array}{c}1 \pm 0 \\
1 \pm 0.8\end{array}$ & $\begin{array}{l}1.5 \pm 1 \\
1.5 \pm 1\end{array}$ & $\begin{array}{l}1 \pm 0 \\
1 \pm 0\end{array}$ \\
\hline Tear osmolarity (mOsm/l) & $312.9 \pm 19.7$ & $314.9 \pm 16.9$ & $344.3 \pm 31$ & $307.6 \pm 16.5$ \\
\hline Phenol red thread test & $21.6 \pm 7.4$ & $17.8 \pm 8.2$ & $21 \pm 5.8$ & $19.4 \pm 9.1$ \\
\hline TBUT & $3.3 \pm 2.4$ & $2.3 \pm 0.9$ & $1.9 \pm 0.3$ & $2.9 \pm 1.7$ \\
\hline Corneal staining (Oxford) & $0 \pm 0$ & $1 \pm 1$ & $1 \pm 0$ & $1 \pm 1$ \\
\hline $\begin{array}{l}\text { Corneal staining (Baylor) } \\
\text { Central } \\
\text { Nasal } \\
\text { Temporal } \\
\text { Superior } \\
\text { Inferior } \\
\text { Total } \\
\end{array}$ & $\begin{array}{c}0 \pm 0 \\
0 \pm 0.8 \\
0 \pm 1 \\
0 \pm 0 \\
0.5 \pm 1 \\
1.4 \pm 1.6\end{array}$ & $\begin{array}{c}0 \pm 0 \\
1 \pm 0.8 \\
1 \pm 0.8 \\
0 \pm 0 \\
1 \pm 0.8 \\
3.5 \pm 3\end{array}$ & $\begin{array}{c}0 \pm 0 \\
1 \pm 0.8 \\
1 \pm 0.8 \\
0 \pm 0 \\
1 \pm 0.8 \\
3.7 \pm 1.8 \\
\end{array}$ & $\begin{aligned} 0 & \pm 0 \\
0 & \pm 1 \\
0 & \pm 0 \\
0 & \pm 0 \\
1 & \pm 1 \\
1.4 & \pm 1.3\end{aligned}$ \\
\hline $\begin{array}{l}\text { Conjunctival staining } \\
\text { Nasal } \\
\text { Temporal } \\
\end{array}$ & $\begin{array}{l}0 \pm 1 \\
0 \pm 1\end{array}$ & $\begin{array}{c}1 \pm 1.5 \\
1 \pm 1\end{array}$ & $\begin{array}{c}1 \pm 0 \\
1 \pm 0.8\end{array}$ & $\begin{array}{l}0 \pm 1 \\
1 \pm 1\end{array}$ \\
\hline Schirmer test & $12.7 \pm 9.4$ & $11.1 \pm 8.4$ & $11.7 \pm 12$ & $13.2 \pm 10.7$ \\
\hline $\begin{array}{l}\text { SIDEQ } \\
\text { Dry eye } \\
\text { Foreign body sensations } \\
\text { Burning } \\
\text { Pain } \\
\text { Itching } \\
\text { Photophobia } \\
\text { Blurred vision } \\
\text { Average } \\
\end{array}$ & $\begin{array}{l}1.1 \pm 1.7 \\
2.2 \pm 2.7 \\
1.4 \pm 2.4 \\
0.9 \pm 1.6 \\
1.4 \pm 2.7 \\
1.1 \pm 2.3 \\
0.7 \pm 1.7 \\
1.3 \pm 1.7\end{array}$ & $\begin{array}{c}0.4 \pm 1 \\
0 \pm 0 \\
0.1 \pm 0.3 \\
0 \pm 0 \\
0 \pm 0 \\
0.2 \pm 0.6 \\
0 \pm 0.1 \\
0.1 \pm 0.2\end{array}$ & $\begin{array}{c}3.5 \pm 2.6 \\
4.1 \pm 2.3 \\
3 \pm 2.2 \\
1.3 \pm 2.4 \\
1.2 \pm 1.6 \\
2.4 \pm 3.4 \\
2 \pm 3.1 \\
2.6 \pm 1.8 \\
\end{array}$ & $\begin{array}{c}2 \pm 1.5 \\
0.4 \pm 0.9 \\
0.4 \pm 0.9 \\
0 \pm 0 \\
1 \pm 1.7 \\
1.6 \pm 3 \\
0.8 \pm 1.1 \\
0.9 \pm 1.2 \\
\end{array}$ \\
\hline OSDI & $22.2 \pm 20$ & $7.6 \pm 12$ & $30.7 \pm 20.5$ & $13.2 \pm 17$ \\
\hline \multicolumn{5}{|c|}{ Molecular tear levels $(\mathrm{pg} / \mathrm{mL})$ detected in at least $80 \%$ of participants } \\
\hline EGF & $1710.7 \pm 1460.5$ & $1649.1 \pm 1277.5$ & $638.8 \pm 618.5$ & $2341.6 \pm 1600.4$ \\
\hline CX3CL1/ Fractalkine & $1166.2 \pm 846.3$ & $980.9 \pm 843.2$ & $355.7 \pm 698.1$ & $1087.6 \pm 877.7$ \\
\hline IL-1Ra & $6649.7 \pm 5386.7$ & $7785 \pm 6618.1$ & $1440.2 \pm 1991.9$ & $14570 \pm 10729.9$ \\
\hline
\end{tabular}




\begin{tabular}{|l|c|c|c|c|}
\hline & $\begin{array}{c}\text { Cluster 1 (n=18) } \\
\text { Mild response }\end{array}$ & $\begin{array}{c}\text { Cluster 2 (n=10) } \\
\text { Corneal epithelial } \\
\text { integrity response }\end{array}$ & $\begin{array}{c}\text { Cluster 3 (n=6) } \\
\text { Tear molecular } \\
\text { response }\end{array}$ & $\begin{array}{c}\text { Cluster 4 (n=5) } \\
\text { Symptomatic } \\
\text { adaptation response }\end{array}$ \\
\hline IL-6 & $64.3 \pm 127.6$ & $38.3 \pm 44.6$ & $79.1 \pm 153.9$ & $41.8 \pm 27$ \\
\hline CXCL8/ IL-8 & $1285.7 \pm 1882.4$ & $503.1 \pm 377.8$ & $106.9 \pm 104.1$ & $923 \pm 636.9$ \\
\hline CXCL10/ IP-10 & $74845.6 \pm 90516.7$ & $29572 \pm 26266$ & $37328.3 \pm 38581.3$ & $50520 \pm 24676.9$ \\
\hline CCL5/ RANTES & $24.9 \pm 11.1$ & $15.3 \pm 11$ & $8.1 \pm 10.1$ & $29.2 \pm 24.1$ \\
\hline VEGF & $975.9 \pm 842.1$ & $401.6 \pm 259$ & $66.7 \pm 21$ & $485.7 \pm 235.5$ \\
\hline MMP-9 & $16763.3 \pm 50780.5$ & $15498.2 \pm 27568.1$ & $696.4 \pm 601.8$ & $3742.6 \pm 6040.3$ \\
\hline
\end{tabular}

$\mathrm{SD}=$ Standard deviation; $\mathrm{IQR}=$ InterQuartile Range $; \mathrm{CI}=$ Confidence interval; TBUT $=$ Tear film

Break-Up Time; SIDEQ = Single-Item Score Dry Eye Questionnaire; OSDI = Ocular Surface

Disease Index; EGF $=$ Epidermal Growth Factor; CX3CL $=$ Chemokine [C-X3-C motif] ligand; IL-1RA = Interleukin-1 Receptor Antagonist; IL-6 = Interleukin-6; CXCL = Chemokine [C-XC motif] ligand; IL-8 = Interleukin-8; IP-10 = interferon- $\gamma$ - Induced Protein-10; CCL = Chemokine [C-C motif] ligand; RANTES = Regulated on Activation, Normal T cell Expressed and Secreted; VEGF $=$ Vascular Endothelial Growth Factor; MMP-9 = matrix metalloproteinase-9. 
Table 3. Predictors for each response profile (cluster). Estimated odds ratio (OR) by the final multivariate logistic regression models. The table shows OR with $95 \%$ confidence intervals. Significant results are denoted in bold. Borderline significant $P$-values $(0.05<P<0.1)$ are denoted in italics. Only controlled adverse desiccating environment (CADE) response prediction variables finally selected in some of the fitted models are shown.

\begin{tabular}{|l|c|c|c|c|}
\hline & $\begin{array}{c}\text { Cluster 1 }(\mathbf{n = 1 8}) \\
\text { Mild response }\end{array}$ & $\begin{array}{c}\text { Cluster 2 }(\mathbf{n}=\mathbf{1 0}) \\
\text { Corneal epithelial } \\
\text { integrity response }\end{array}$ & $\begin{array}{c}\text { Cluster } \mathbf{3}(\mathbf{n}=\mathbf{6}) \\
\text { Tear molecular } \\
\text { response }\end{array}$ & $\begin{array}{c}\text { Cluster 4 (n=5) } \\
\text { Symptomatic } \\
\text { adaptation } \\
\text { response }\end{array}$ \\
\hline Corneal staining (Oxford) & $\mathbf{0 . 0 8 ( 0 . 0 1 ; 0 . 5 7 )}$ & - & - & - \\
\hline Temporal corneal staining & - & $\mathbf{1 2 . 6 5 ( 1 . 3 8 ; 1 1 5 . 8 5 )}$ & - & - \\
\hline Nasal conjunctival staining & $0.2(0.04 ; 1.06)$ & - & - & - \\
\hline SIDEQ. Average & - & $0.09(0.01 ; 1.21)$ & - & - \\
\hline IL-1Ra & - & - & - & $3.08(0.9 ; 10.5)$ \\
\hline CXCL10/ IP-10 & $\mathbf{2 . 2 ( 1 . 0 9 ; 4 . 4 2 )}$ & $\mathbf{0 . 2 6}(\mathbf{0 . 0 7} \mathbf{0 . 9 1})$ & - & - \\
\hline VEGF & & - & $0.01(0 ; 1.18)$ & - \\
\hline
\end{tabular}

SIDEQ $=$ Single-Item Score Dry Eye Questionnaire; IL-1RA = InterLeukin-1 Receptor

Antagonist; $\mathrm{CXCL}=$ Chemokine $[\mathrm{C}-\mathrm{X}-\mathrm{C}$ motif] ligand; IP-10 = interferon- $\gamma$ - Induced Protein10; VEGF = Vascular Endothelial Growth Factor.

Table 4. Discrimination ability of the final multivariate logistic regression models. Area under the curve (AUC), sensibility and specificity values based on leave-one-out-cross-validation (LOOCV) procedure, are shown. AUC values statistically different from 0.5 (random chance) are denoted in bold.

\begin{tabular}{|c|c|c|c|}
\hline & $\begin{array}{c}\text { AUC } \\
\mathbf{( 9 5 \%} \mathbf{C I})\end{array}$ & $\begin{array}{c}\text { Sensitivity (\%) } \\
\mathbf{( 9 5 \%} \mathbf{C I})\end{array}$ & $\begin{array}{c}\text { Specificity (\%) } \\
\mathbf{( 9 5 \%} \mathbf{C I})\end{array}$ \\
\hline Cluster 1 (n=18) & $\mathbf{0 . 8 1 7 5}$ & 83.33 & 71.43 \\
Mild response & $\mathbf{( 0 . 6 8 0 9 ; 0 . 9 5 4 )}$ & $(66.12 ; 100)$ & $(52.11 ; 90.75)$ \\
\hline Cluster 2 (n=10) & $\mathbf{0 . 8 7 9 3}$ & 90.00 & 96.55 \\
Corneal epithelial integrity response & $\mathbf{( 0 . 6 8 9 ;} \mathbf{1})$ & $(71.41 ; 100)$ & $(89.91 ; 100)$ \\
\hline Cluster 3 (n=6) & $\mathbf{0 . 9 5 4 5}$ & 100.00 & 87.88 \\
Tear molecular response & $\mathbf{( 0 . 8 9 1 ;} \mathbf{1})$ & $(87 ; 100)$ & $(76.74 ; 99.01)$ \\
\hline Cluster 4 $(\mathbf{n}=\mathbf{5})$ & $\mathbf{0 . 7 3 5 3}$ & 80.00 & 67.65 \\
Symptomatic adaptation response & $\mathbf{( 0 . 5 4 4 ; 0 . 9 2 6 2 )}$ & $(44.94 ; 100)$ & $(51.92 ; 83.37)$ \\
\hline
\end{tabular}

AUC $=$ area under the curve; $\mathrm{CI}=$ confidence interval; LOOCV $=$ leave-one-out-crossvalidation 


\section{FIGURE LEGENDS}

Figure 1. Clinical response profiles to controlled adverse desiccating environment (CADE) for each of the four clusters found in CADE effect dataset by trimmed $k$-means clustering with $k=4$ and $\alpha=0.025$. The $Y$-axis represents the relative change (percentage) between pre-exposure and postexposure values. Each of the equi-spaced vertical ticks on X-axis represents a different clinical variable in CADE effect dataset. A different solid grey line for each participant is plotted. Solid black lines and circles represent the average response profile. Shaded area indicates the 95\% confidence intervals for the mean constructed using bootstrap procedure based on 5000 replications. Increase in corneal staining and decrease in tear break-up time occurs across the four clusters, in contrast, subjective change is only clearly manifested in cluster 4 . 
Figure 2. Molecular response profiles to controlled adverse desiccating environment (CADE) of each of 4 cluster found in CADE effect dataset by trimmed $\mathbf{k}$-means clustering with $\mathbf{k}=\mathbf{4}$ and $\boldsymbol{\alpha}=\mathbf{0 . 0 2 5}$. The $Y$-axis represents the log2-Fold change from pre- to post-CADE exposure. Each of the equispaced vertical ticks on X-axis represents a different detected cytokine in CADE effect data set. A different grey line for each subject is plotted. Solid black lines and circles represent the average response profile. Shaded area indicates the 95\% confidence intervals for the mean constructed using bootstrap procedure based on 5000 replications. A modest but significant unbalance of tear inflammatory biomarkers should be expected, except for some individuals (cluster 3) who might show and overwhelming response. 
Figure 3. Association between each controlled adverse desiccation environment (CADE) response prediction variable and the response profiles (clusters). The $x$-axis is the base-2 logarithmic odds ratio (log2 OR) estimated by one-vs-all binary logistic regression analysis. Black circles and triangles indicate statistically significant associations at $5 \%$ and $10 \%$ significance levels, respectively. White small circles indicate no significant associations at $10 \%$ level. The $95 \%$ confidence intervals for log2 OR are plotted as horizontal lines. The vertical bold line represents the no association value. For each CADE response prediction variable, positive values (right to the vertical line) mean positive association between CADE response prediction variable and cluster membership, while negative values (left to the vertical line) mean negative association. Clinical and tear molecule variables that characterize each cluster might not be the same ones that can predict the response of each cluster. 
Figure 4. Summary of the statistical procedure performed and sequential outcomes obtained. LOD = Limits of detection; CADE = Controlled adverse desiccating environment; $\mathrm{PCA}=$ Principal component analysis; $\mathrm{PC}=$ Principal component; $\mathrm{Cl}=$ Cluster . 
Cluster 1: Mild response ( $\mathrm{n}=18)$

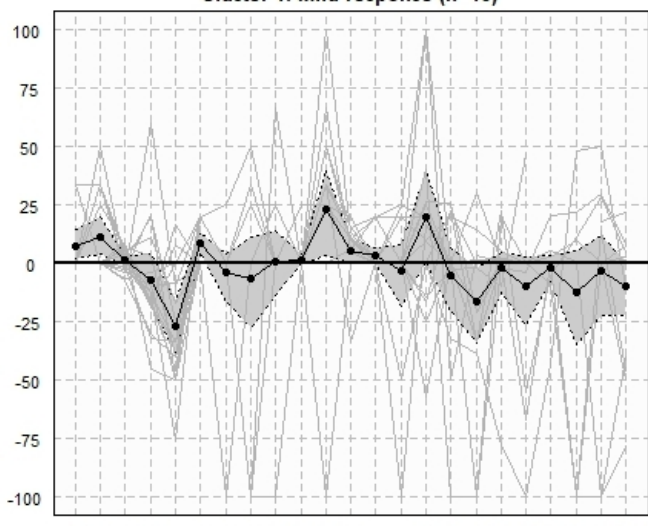

ำ 㐫

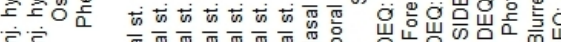

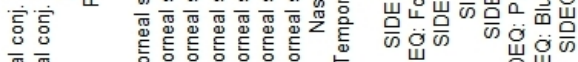

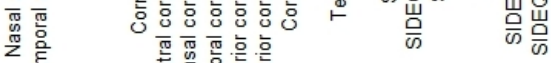

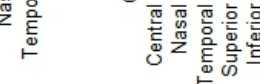

Cluster 2: Corneal epithelial integrity response $(n=10)$

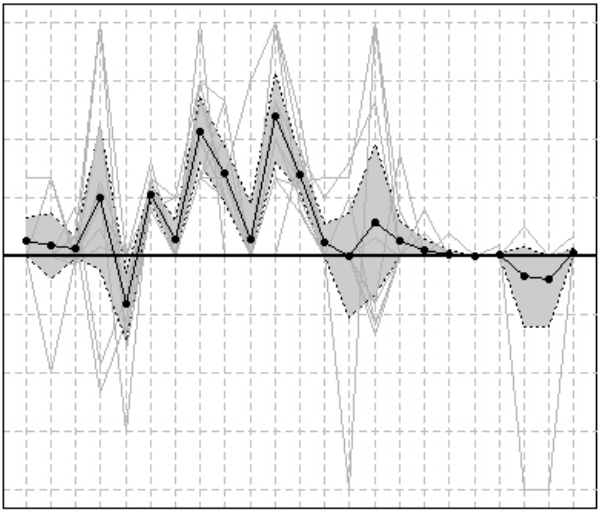

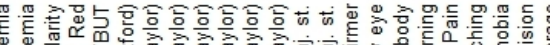

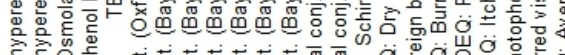

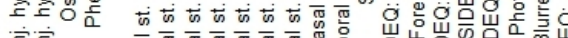
言高

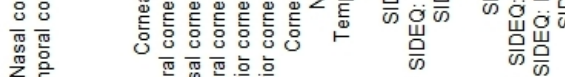

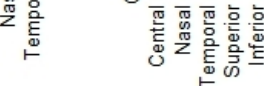

Cluster 3: Tear molecular response $(\mathrm{n}=6)$

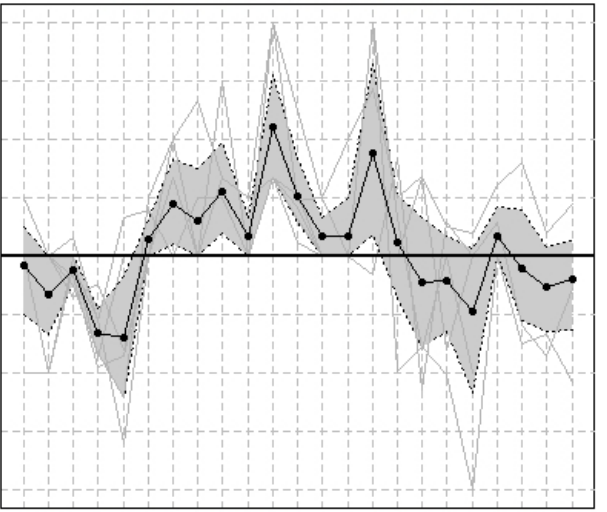

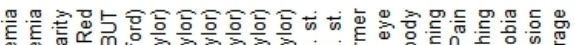

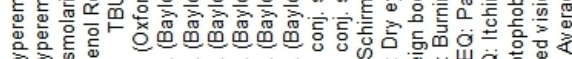

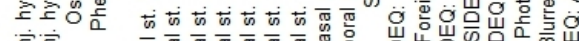

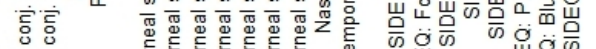

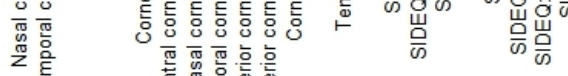

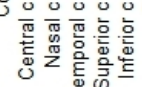

Cluster 4: Symptomatic adaptation response $(\mathrm{n}=5)$

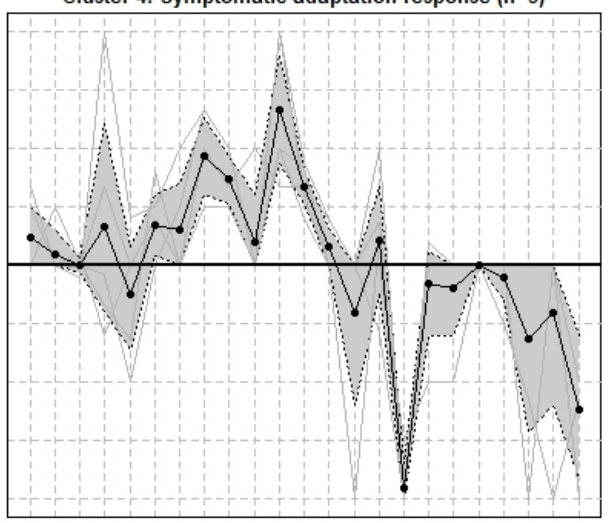

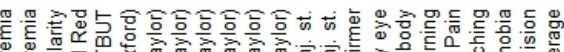

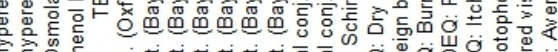

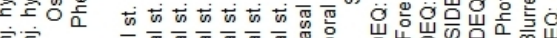

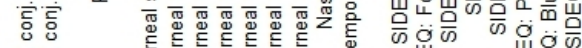

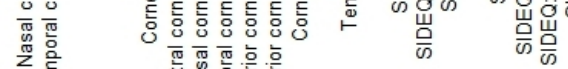

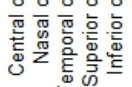



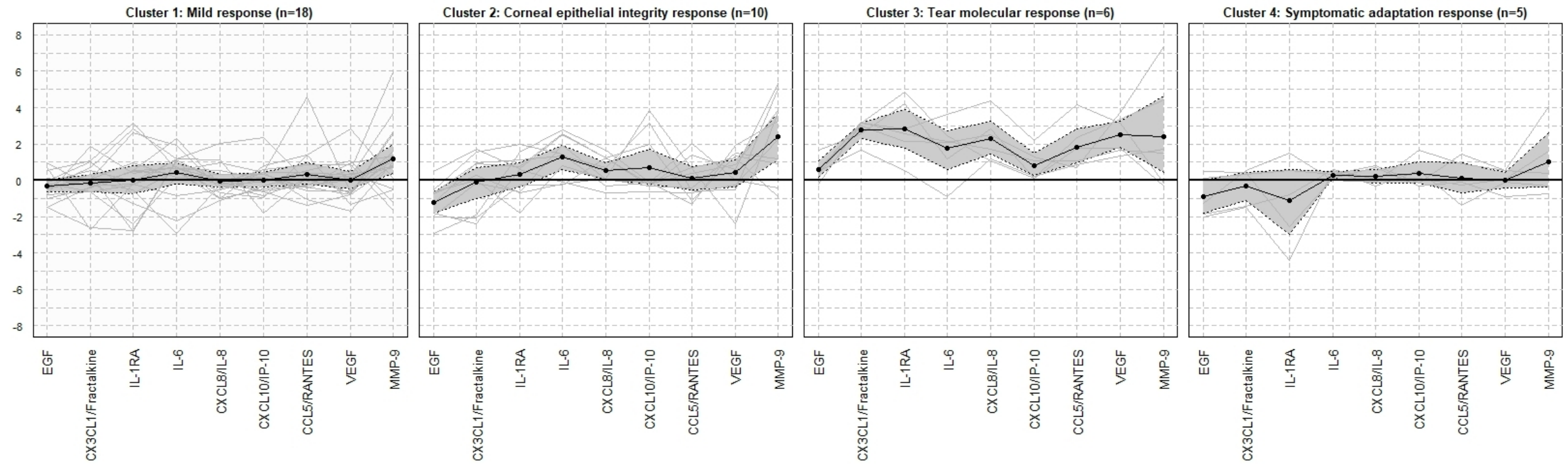


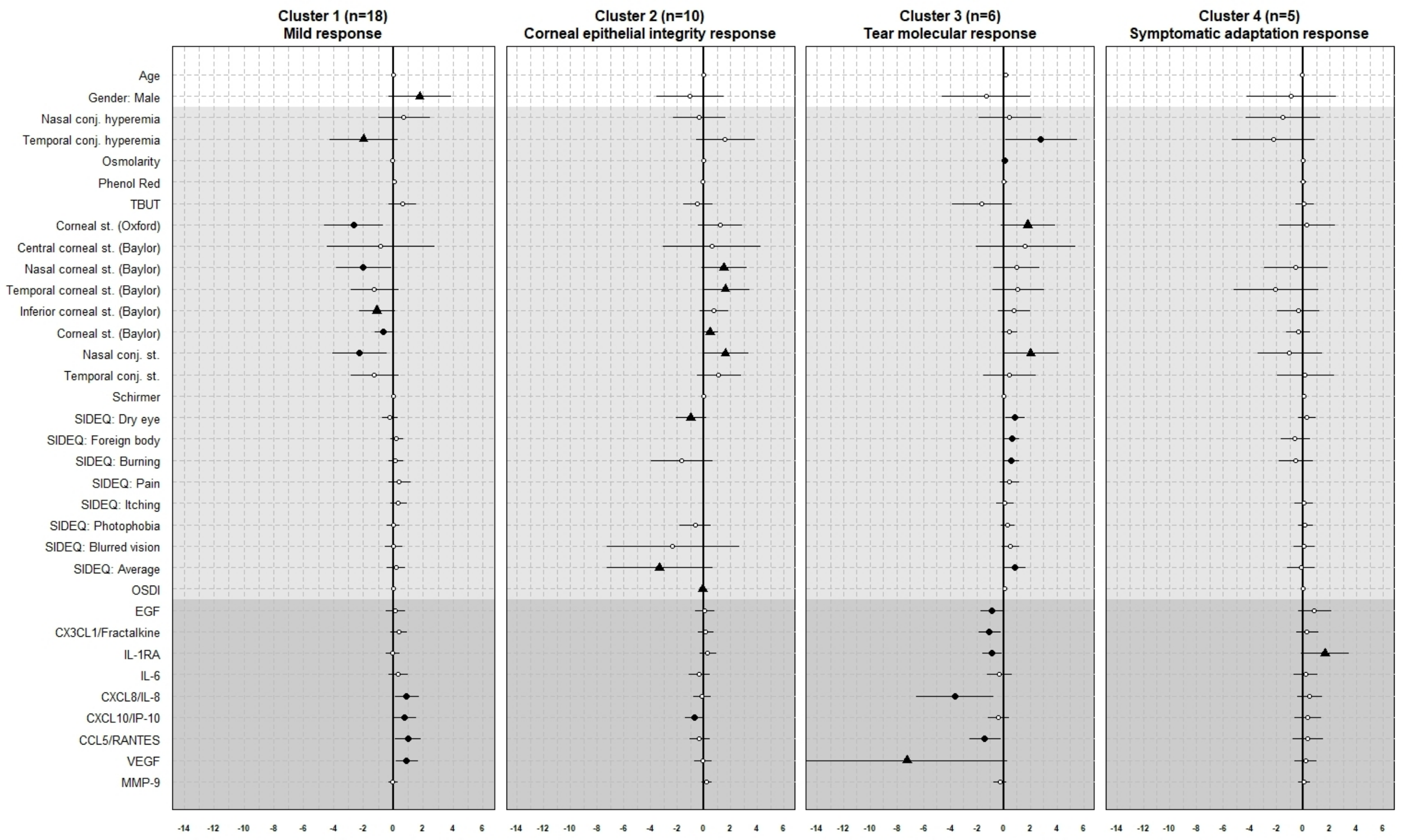




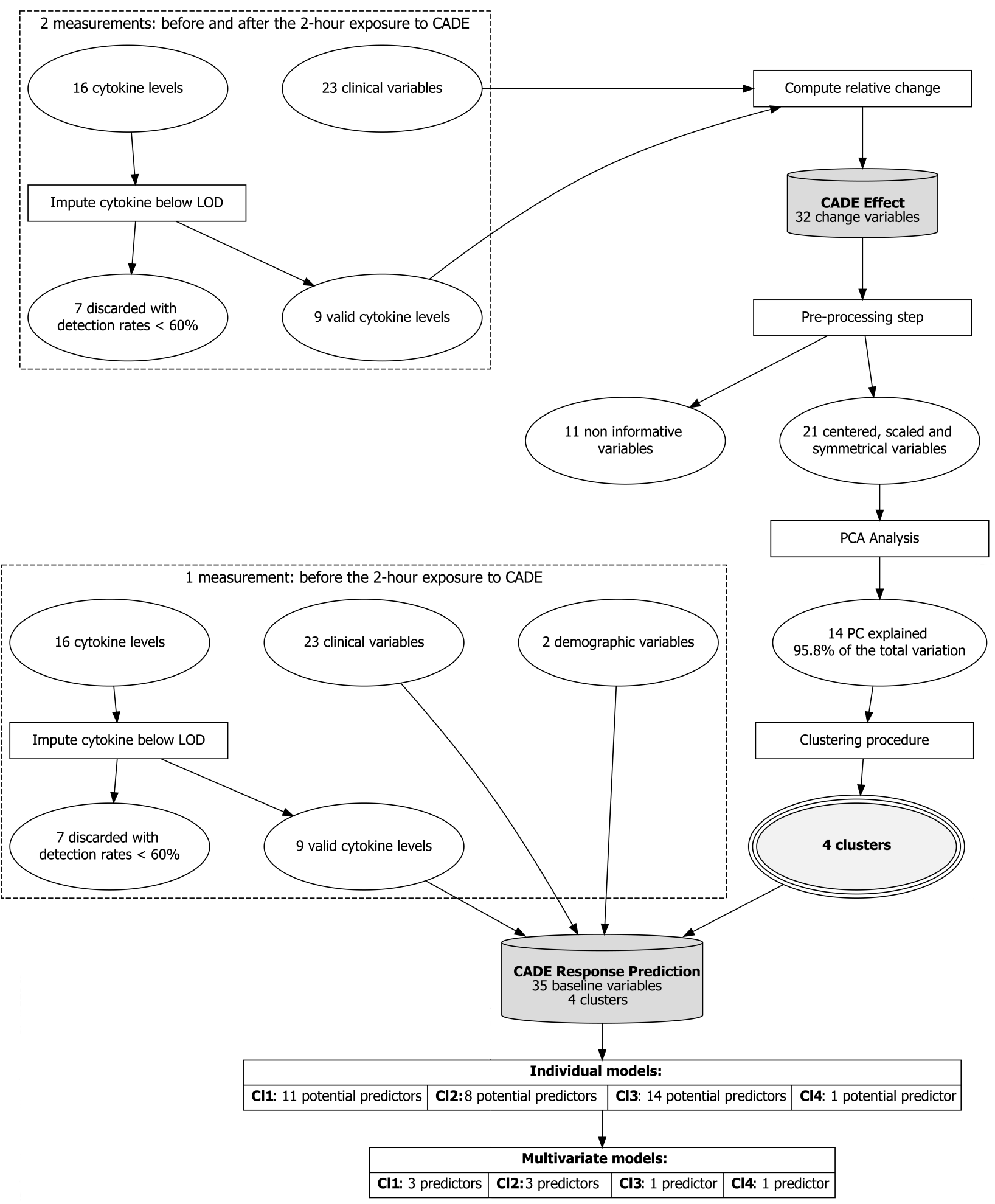




\section{APPENDIX A}

Table A1. Limit and percentage of detection of the 16 tear molecules analyzed in tear samples.

\begin{tabular}{|c|c|c|c|}
\hline & \multirow{2}{*}{$\begin{array}{l}\text { Limit of } \\
\text { detection } \\
(\mathrm{pg} / \mathrm{ml})\end{array}$} & \multicolumn{2}{|c|}{$\begin{array}{c}\text { Rate of detection (\%) } \\
(95 \% \mathrm{Cl})\end{array}$} \\
\hline & & Pre-exposure & Post-exposure \\
\hline EGF & 27 & $\begin{array}{c}95 \\
(81.79 ; 99.13)\end{array}$ & $\begin{array}{c}95 \\
(81.79 ; 99.13)\end{array}$ \\
\hline $\begin{array}{c}\text { CX3CL1/ } \\
\text { Fractalkine }\end{array}$ & 60 & $\begin{array}{c}85 \\
(69.48 ; 93.75) \\
\end{array}$ & $\begin{array}{c}92.5 \\
(78.52 ; 98.04) \\
\end{array}$ \\
\hline IFN-g & 1 & $\begin{array}{c}0 \\
(0 ; 10.91)\end{array}$ & $\begin{array}{c}0 \\
(0 ; 10.91)\end{array}$ \\
\hline IL-1b & 4 & $\begin{array}{c}30 \\
(17.09 ; 46.71)\end{array}$ & $\begin{array}{c}20 \\
(9.62 ; 36.14)\end{array}$ \\
\hline IL-1Ra & 29 & $\begin{array}{c}100 \\
(89.09 ; 100)\end{array}$ & $\begin{array}{c}100 \\
(89.09 ; 100)\end{array}$ \\
\hline IL-2 & 3 & $\begin{array}{c}20 \\
(9.62 ; 36.14)\end{array}$ & $\begin{array}{c}25 \\
(13.25 ; 41.52)\end{array}$ \\
\hline IL-6 & 3 & $\begin{array}{c}82.5 \\
(66.64 ; 92.11)\end{array}$ & $\begin{array}{c}95 \\
(81.79 ; 99.13)\end{array}$ \\
\hline CXCL8/ IL-8 & 2 & $\begin{array}{c}100 \\
(89.09 ; 100)\end{array}$ & $\begin{array}{c}97.5 \\
(85.27 ; 99.87) \\
\end{array}$ \\
\hline IL-10 & 3 & $\begin{array}{c}7.5 \\
(1.96 ; 21.48)\end{array}$ & $\begin{array}{c}20 \\
(9.62 ; 36.14)\end{array}$ \\
\hline IL-12p70 & 4 & $\begin{array}{c}2.5 \\
(0.13 ; 14.73)\end{array}$ & $\begin{array}{c}0 \\
(0 ; 10.91)\end{array}$ \\
\hline IL-17A & 2 & $\begin{array}{c}0 \\
(0 ; 10.91) \\
\end{array}$ & $\begin{array}{c}0 \\
(0 ; 10.91)\end{array}$ \\
\hline CXCL10/ IP-10 & 12 & $\begin{array}{c}92.5 \\
(78.52 ; 98.04)\end{array}$ & $\begin{array}{c}95 \\
(81.79 ; 99.13)\end{array}$ \\
\hline CCL5/ RANTES & 10 & $\begin{array}{c}87.5 \\
(72.4 ; 95.31)\end{array}$ & $\begin{array}{c}90 \\
(75.4 ; 96.75)\end{array}$ \\
\hline TNF-a & 1 & $\begin{array}{c}2.5 \\
(0.13 ; 14.73)\end{array}$ & $\begin{array}{c}7.5 \\
(1.96 ; 21.48)\end{array}$ \\
\hline VEGF & 58 & $\begin{array}{c}77.5 \\
(61.15 ; 88.6)\end{array}$ & $\begin{array}{c}85 \\
(69.48 ; 93.75)\end{array}$ \\
\hline MMP-9 & 10 & $\begin{array}{c}87.5 \\
(72.4 ; 95.31)\end{array}$ & $\begin{array}{c}90 \\
(75.4 ; 96.75)\end{array}$ \\
\hline
\end{tabular}

$\mathrm{Cl}=$ Confidence interval; $\mathrm{EGF}=$ Epidermal Growth Factor; $\mathrm{CX} 3 \mathrm{CL}=$ Chemokine $[\mathrm{C}-\mathrm{X} 3-\mathrm{C}$ motif] ligand; IFN-g = interferon - g; IL-1b= Interleukin-1b;IL-1RA = Interleukin-1 Receptor Antagonist; IL-2 = Interleukin-2; IL-6 = Interleukin-6; CXCL = Chemokine [C-X-C motif] ligand; IL-8 = Interleukin8; IL-10 = Interleukin-10; IL-12p70 = Interleukin-12p70; IL-17A = Interleukin-17A; IP-10 = interferon- gamma- Induced Protein-10; $\mathrm{CCL}=$ Chemokine [C-C motif] ligand; RANTES = Regulated on Activation, Normal T cell Expressed and Secreted; TNF-a = tumor necrosis factor a; VEGF = Vascular Endothelial Growth Factor; MMP-9 = matrix metalloproteinase-9. 
Table A2. Results of the principal component analysis (PCA) for condensing the 21 informative controlled adverse desiccating environment (CADE) effect variables into 14 statistically-independent dimensions. Since skewness and the magnitude of the variables influence the PCA results, each of the original variables was previously centered, scaled and applied a Box and Cox transformation. The table shows the contribution of each CADE effect variable to selected principal components (PCs).

\begin{tabular}{|c|c|c|c|c|c|c|c|c|c|c|c|c|c|c|}
\hline & PC1 & PC2 & PC3 & PC4 & PC5 & PC6 & $\mathrm{PC7}$ & PC8 & PC9 & PC10 & PC11 & PC12 & PC13 & PC14 \\
\hline Tear osmolarity & -0.10 & 0.02 & -0.29 & 0.59 & -0.01 & 0.10 & -0.18 & 0.30 & -0.11 & 0.04 & -0.25 & 0.22 & -0.16 & 0.07 \\
\hline $\begin{array}{l}\text { Phenol Red } \\
\text { Thread Test }\end{array}$ & -0.11 & 0.21 & 0.22 & -0.21 & 0.34 & -0.32 & -0.07 & 0.39 & -0.25 & -0.26 & -0.15 & -0.12 & -0.08 & 0.17 \\
\hline TBUT & -0.24 & 0.08 & -0.07 & -0.12 & 0.21 & 0.47 & 0.23 & 0.23 & -0.09 & -0.25 & 0.26 & -0.41 & 0.01 & -0.05 \\
\hline $\begin{array}{r}\text { Corneal staining } \\
\text { (Oxford) }\end{array}$ & 0.07 & 0.31 & 0.13 & 0.24 & 0.22 & 0.01 & -0.43 & 0.25 & 0.42 & 0.14 & 0.11 & -0.23 & -0.04 & 0.06 \\
\hline $\begin{array}{c}\text { Corneal staining } \\
\text { (Baylor) }\end{array}$ & & & & & & & & & & & & & & \\
\hline Nasal & 0.02 & 0.42 & -0.04 & 0.06 & 0.14 & -0.13 & -0.04 & -0.16 & -0.38 & 0.47 & 0.40 & 0.14 & -0.08 & 0.05 \\
\hline Temporal & 0.03 & 0.43 & -0.20 & -0.08 & -0.14 & 0.00 & 0.15 & -0.15 & -0.04 & -0.36 & -0.33 & 0.08 & -0.36 & -0.14 \\
\hline Inferior & 0.13 & 0.31 & 0.21 & -0.22 & -0.33 & 0.13 & -0.23 & -0.10 & 0.29 & -0.03 & -0.22 & -0.06 & 0.37 & 0.19 \\
\hline Total & 0.09 & 0.52 & 0.00 & -0.03 & -0.02 & 0.03 & 0.22 & -0.06 & 0.11 & -0.05 & 0.14 & 0.11 & -0.13 & 0.01 \\
\hline Schirmer test & 0.05 & 0.04 & -0.05 & 0.53 & -0.09 & -0.46 & 0.29 & -0.16 & 0.14 & -0.31 & 0.19 & -0.34 & 0.23 & 0.09 \\
\hline SIDEQ & & & & & & & & & & & & & & \\
\hline Dry eye & 0.04 & -0.08 & -0.45 & -0.31 & 0.08 & -0.34 & -0.11 & -0.02 & 0.33 & 0.05 & 0.04 & 0.06 & -0.24 & 0.07 \\
\hline $\begin{array}{r}\text { Foreign body } \\
\text { sensations }\end{array}$ & -0.05 & 0.22 & -0.39 & 0.03 & -0.31 & 0.14 & -0.29 & 0.00 & -0.34 & 0.03 & -0.10 & -0.28 & 0.28 & -0.06 \\
\hline Average & -0.03 & -0.05 & -0.53 & -0.22 & -0.03 & -0.17 & -0.16 & 0.21 & 0.08 & -0.14 & 0.22 & -0.04 & 0.17 & -0.07 \\
\hline EGF & 0.32 & -0.22 & 0.12 & -0.04 & -0.06 & -0.06 & -0.28 & 0.06 & -0.33 & -0.08 & 0.02 & -0.17 & -0.06 & -0.10 \\
\hline $\begin{array}{r}\text { CX3CL1/ } \\
\text { Fractalkine }\end{array}$ & 0.37 & -0.02 & -0.03 & -0.12 & -0.15 & -0.03 & 0.05 & 0.00 & -0.24 & -0.10 & 0.31 & 0.02 & 0.01 & 0.47 \\
\hline IL-1Ra & 0.35 & -0.11 & -0.10 & 0.10 & -0.03 & 0.25 & 0.06 & 0.11 & 0.01 & -0.11 & -0.12 & -0.01 & -0.24 & 0.52 \\
\hline IL-6 & 0.27 & 0.09 & -0.13 & -0.03 & 0.17 & 0.07 & 0.35 & 0.44 & 0.09 & 0.11 & -0.05 & 0.36 & 0.48 & -0.03 \\
\hline CXCL8/ IL-8 & 0.37 & 0.03 & -0.09 & 0.06 & 0.19 & 0.00 & 0.11 & -0.13 & -0.14 & -0.04 & -0.17 & 0.00 & 0.19 & -0.37 \\
\hline CXCL10/ IP-10 & 0.31 & 0.04 & 0.11 & 0.05 & 0.27 & -0.08 & -0.34 & -0.07 & -0.06 & -0.39 & 0.06 & 0.20 & 0.05 & -0.23 \\
\hline CCL5/ RANTES & 0.31 & -0.03 & 0.05 & 0.05 & -0.29 & 0.21 & 0.02 & 0.22 & 0.19 & -0.01 & 0.37 & -0.06 & -0.32 & -0.40 \\
\hline VEGF & 0.32 & 0.02 & -0.06 & -0.07 & 0.11 & -0.15 & 0.21 & 0.08 & -0.01 & 0.43 & -0.35 & -0.52 & -0.14 & -0.07 \\
\hline MMP-9 & 0.09 & -0.05 & -0.23 & 0.03 & 0.52 & 0.35 & -0.09 & -0.48 & 0.12 & -0.03 & 0.02 & -0.09 & 0.05 & 0.13 \\
\hline $\begin{array}{c}\text { Variance } \\
\text { explained (\%) }\end{array}$ & 25.49 & 14.64 & 11.64 & 7.00 & 6.64 & 5.60 & 5.04 & 4.47 & 3.96 & 3.29 & 2.50 & 2.23 & 2.01 & 1.32 \\
\hline $\begin{array}{c}\text { Cumulative } \\
\text { variance } \\
\text { explained (\%) }\end{array}$ & 25.49 & 40.13 & 51.77 & 58.77 & 65.41 & 71.00 & 76.04 & 80.51 & 84.47 & 87.76 & 90.26 & 92.49 & 94.50 & 95.83 \\
\hline
\end{tabular}


Table A3. Clinical and molecular response profiles to controlled adverse desiccating environment (CADE) for each of the 4 clusters found in CADE effect dataset by trimmed kmeans clustering with $k=4$ and $\alpha=0.025$. Mean and $95 \%$ confidence intervals $(\mathrm{Cl})$ for the mean of all CADE effect variables are shown for each cluster. Confidence intervals are constructed using bootstrap procedure based on 5000 replications. Italic font indicates statistical difference from 0 at the 0.05 level (the $95 \% \mathrm{Cl}$ does not contain zero value). Relevant changes are shown in boldface. Statistically significant changes in clinical parameters greater than $25 \%$ were considered relevant changes. For tear molecule levels, this threshold was established at 2-fold (1 log2-FC).

\begin{tabular}{|c|c|c|c|c|c|}
\hline & $\begin{array}{l}\text { Cluster } 1(\mathrm{n}=18) \\
\text { Mild response }\end{array}$ & $\begin{array}{l}\text { Cluster } 2(n=10) \\
\text { Corneal epithelial } \\
\text { integrity response }\end{array}$ & $\begin{array}{l}\text { Cluster } \mathbf{3}(\mathrm{n}=6) \\
\text { Tear molecular } \\
\text { response }\end{array}$ & $\begin{array}{c}\text { Cluster } 4(\mathrm{n}=5) \\
\text { Symptomatic } \\
\text { adaptation response }\end{array}$ & $\begin{array}{c}\text { Trimmed } \\
\text { observations } \\
(n=1)\end{array}$ \\
\hline \multicolumn{6}{|c|}{$\begin{array}{l}\text { Clinical parameters } \\
\text { (relative change from pre- exposure in \%) }\end{array}$} \\
\hline \multicolumn{6}{|l|}{ Conjunctival hyperemia } \\
\hline Nasal & $7.4 \%(1.9 \% ; 14.8 \%)$ & $6.7 \%(0 \% ; 16.7 \%)$ & $\begin{array}{c}-4.2 \% \\
(-25 \% ; 12.5 \%)\end{array}$ & $11.7 \%(0 \% ; 25 \%)$ & $0 \%$ \\
\hline Temporal & $11.6 \%$ (3.7\%;19.9\%) & $5 \%(-11.7 \% ; 20 \%)$ & $\begin{array}{c}-16.7 \% \\
(-33.3 \% ; 0 \%) \\
\end{array}$ & $5 \%(0 \% ; 15 \%)$ & $0 \%$ \\
\hline Tear osmolarity & $1.5 \%(-0.7 \% ; 3.6 \%)$ & $3.2 \%(-0.7 \% ; 8 \%)$ & $-6 \%(-12.2 \% ; 0.9 \%)$ & $0.3 \%(-2.9 \% ; 3.3 \%)$ & $-2.3 \%$ \\
\hline Phenol red thread test & $\begin{array}{c}-7.1 \% \\
(-17 \% ; 4.4 \%) \\
\end{array}$ & $\begin{array}{c}24.9 \% \\
(-6.8 \% ; 55.3 \%) \\
\end{array}$ & $\begin{array}{c}-32.9 \% \\
(-41.8 \% ;-22.7 \%) \\
\end{array}$ & $\begin{array}{c}16.4 \% \\
(-19.7 \% ; 60.7 \%) \\
\end{array}$ & $23.8 \%$ \\
\hline TBUT & $\begin{array}{c}-27.1 \% \\
(-37.6 \% ;-16.2 \%)\end{array}$ & $\begin{array}{c}-20.4 \% \\
(-35.7 \% ;-7.8 \%)\end{array}$ & $\begin{array}{c}-34.4 \% \\
(-60.6 \% ;-7.8 \%)\end{array}$ & $\begin{array}{c}-12.2 \% \\
(-36.3 \% ; 8.2 \%) \\
\end{array}$ & $75.2 \%$ \\
\hline Corneal staining (Oxford) & $8.9 \%(4.4 \% ; 13.3 \%)$ & $26.7 \%$ (22.8\%;30.8\%) & $7.5 \%(0 \% ; 15.8 \%)$ & $17 \%(4 \% ; 30 \%)$ & $0 \%$ \\
\hline \multicolumn{6}{|l|}{ Corneal staining (Baylor) } \\
\hline Central & $-4.2 \%(-16.7 \% ; 4.2 \%)$ & $7.5 \%(0 \% ; 15 \%)$ & $22.2 \%(5.6 \% ; 38.9 \%)$ & $15 \%(0 \% ; 35 \%)$ & $33.3 \%$ \\
\hline Nasal & $-6.5 \%(-27.8 \% ; 12.5 \%)$ & $53.3 \%(40 \% ; 69.2 \%)$ & $15.3 \%(0 \% ; 37.5 \%)$ & $46.7 \%(30 \% ; 63.3 \%)$ & $33.3 \%$ \\
\hline Temporal & $0.9 \%(-13.9 \% ; 13.9 \%)$ & $35.8 \%$ (24.2\%;47.5\%) & $27.8 \%(9.7 \% ; 48.6 \%)$ & $36.7 \%$ (26.7\%;46.7\%) & $33.3 \%$ \\
\hline Superior & $1.4 \%(0 \% ; 4.2 \%)$ & $7.5 \%(0 \% ; 22.5 \%)$ & $8.3 \%(0 \% ; 16.7 \%)$ & $10 \%(0 \% ; 30 \%)$ & $25 \%$ \\
\hline Inferior & $23.1 \%(3.7 \% ; 40.3 \%)$ & $60 \%(40 \% ; 80 \%)$ & $\begin{array}{c}55.6 \% \\
(33.3 \% ; 77.8 \%)\end{array}$ & $66.7 \%(43.3 \% ; 90 \%)$ & $0 \%$ \\
\hline Total & $5.6 \%(-0.2 \% ; 9.9 \%)$ & $34.9 \%$ (27.6\%;43.1\%) & $26 \%(14.0 \% ; 41.7 \%)$ & $33.8 \%$ (26.2\%;40.9\%) & $30.8 \%$ \\
\hline \multicolumn{6}{|l|}{ Conjunctival staining } \\
\hline Nasal & $3.3 \%(0 \% ; 6.7 \%)$ & $5.8 \%(0 \% ; 14.2 \%)$ & $8.3 \%(0 \% ; 16.7 \%)$ & $8 \%(0 \% ; 16 \%)$ & $0 \%$ \\
\hline Temporal & $\begin{array}{c}-3.3 \% \\
(-18.3 \% ; 7.8 \%)\end{array}$ & $\begin{array}{c}-0.2 \% \\
(-26.7 \% ; 18.8 \%)\end{array}$ & $\begin{array}{c}8.3 \% \\
(0 \% ; 25 \%)\end{array}$ & $\begin{array}{c}-20 \% \\
(-60 \% ; 0 \%)\end{array}$ & $0 \%$ \\
\hline Schirmer test & $19.7 \%(1.2 \% ; 39.3 \%)$ & $\begin{array}{c}14.4 \%(- \\
14.6 \% ; 46.8 \%) \\
\end{array}$ & $\begin{array}{c}44 \% \\
(9.3 \% ; 78.6 \%)\end{array}$ & $\begin{array}{c}10.5 \% \\
(-11.4 \% ; 33.6 \%) \\
\end{array}$ & $30 \%$ \\
\hline \multicolumn{6}{|l|}{ SIDEQ } \\
\hline Dry eye & $\begin{array}{c}-5.2 \% \\
(-20 \% ; 6.5 \%)\end{array}$ & $\begin{array}{c}6.3 \% \\
(0 \% ; 17.1 \%)\end{array}$ & $\begin{array}{c}5.7 \% \\
(-20.5 \% ; 25.2 \%)\end{array}$ & $\begin{array}{c}-95 \% \\
(-100 \% ;-85 \%)\end{array}$ & $0 \%$ \\
\hline $\begin{array}{l}\text { Foreign body } \\
\text { sensations }\end{array}$ & $\begin{array}{c}-16.5 \% \\
(-33.2 \% ; 0 \%)\end{array}$ & $\begin{array}{c}3 \% \\
(0 \% ; 7 \%)\end{array}$ & $\begin{array}{c}-11.4 \% \\
(-38.8 \% ; 16.3 \%)\end{array}$ & $\begin{array}{c}-8 \% \\
(-30 \% ; 6 \%)\end{array}$ & $0 \%$ \\
\hline Burning & $\begin{array}{c}-2 \% \\
(-12.5 \% ; 5.2 \%)\end{array}$ & $\begin{array}{c}1 \% \\
(0 \% ; 3 \%)\end{array}$ & $\begin{array}{c}-10.7 \% \\
(-32.1 \% ; 8.6 \%)\end{array}$ & $\begin{array}{c}-10 \% \\
(-30 \% ; 0 \%)\end{array}$ & $0 \%$ \\
\hline Pain & $-9.9 \%$ & $0 \%$ & $-23.3 \%$ & $0 \%$ & $0 \%$ \\
\hline
\end{tabular}




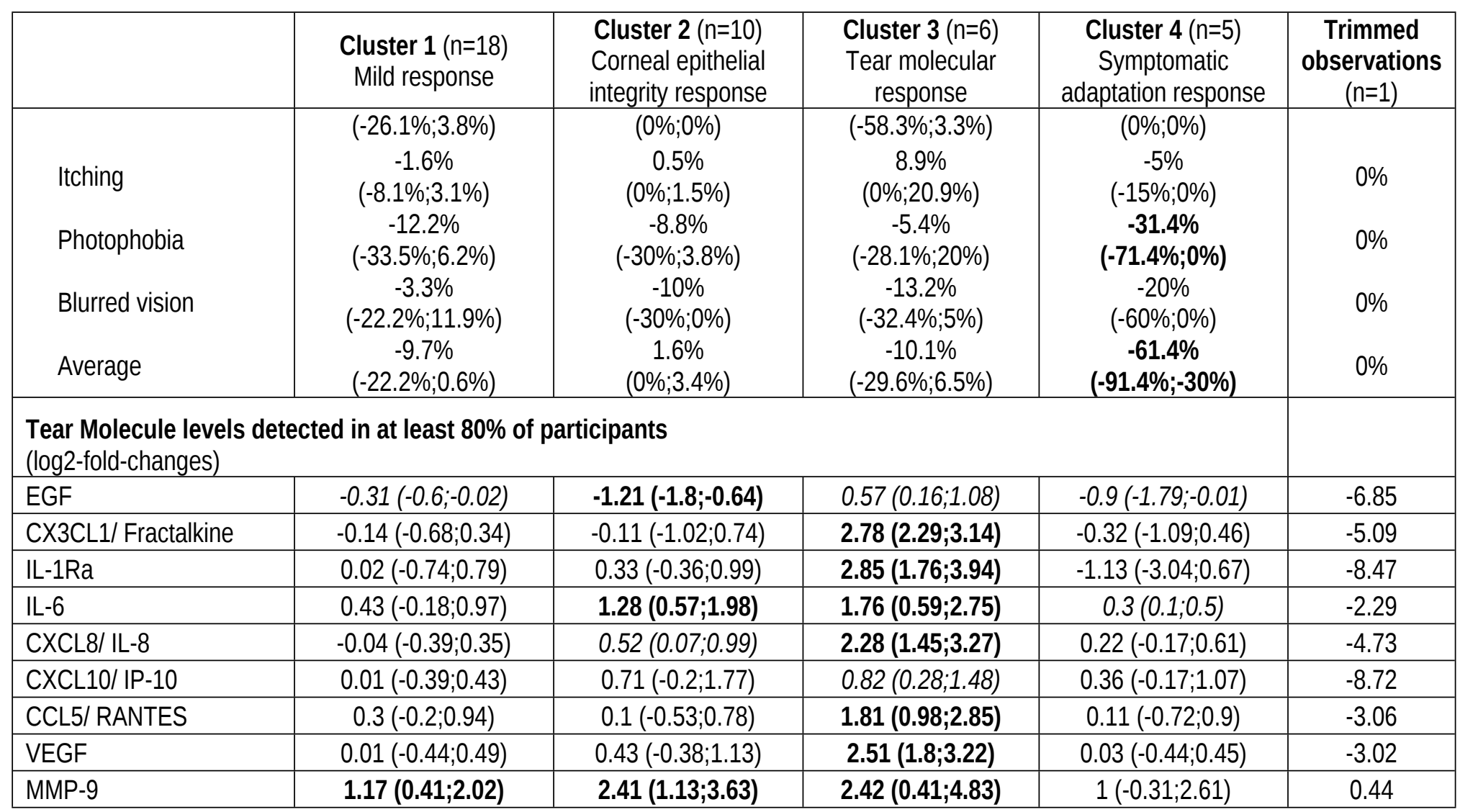

$\mathrm{Cl}=$ Confidence interval; TBUT = Tear film Break-Up Time; SIDEQ = Single-ltem Score Dry Eye Questionnaire; EGF = Epidermal Growth Factor; CX3CL = Chemokine [C-X3-C motif] ligand; IL1RA = Interleukin-1 Receptor Antagonist; IL-6 = Interleukin-6; CXCL = Chemokine [C-X-C motif] ligand; IL-8 = Interleukin-8; IP-10 = interferon- gamma- Induced Protein-10; CCL = Chemokine [CC motif] ligand; RANTES $=$ Regulated on Activation, Normal T cell Expressed and Secreted; VEGF = Vascular Endothelial Growth Factor; MMP-9 = matrix metalloproteinase-9. 
Table A4. Summary of the exhaustive search performed to find the best multivariate classifiers. Multivariate logistic regression models of response profiles with optimal Bayesian information criterion (BIC) by number of predictors. Mk represents the model of size k, that is, based on k predictors (i.e. M1, M2, etc). Better model by size is the one with the lower $\mathrm{BIC}$ and it is highlighted with a grey-shaded area.

Cluster 1: Mild response

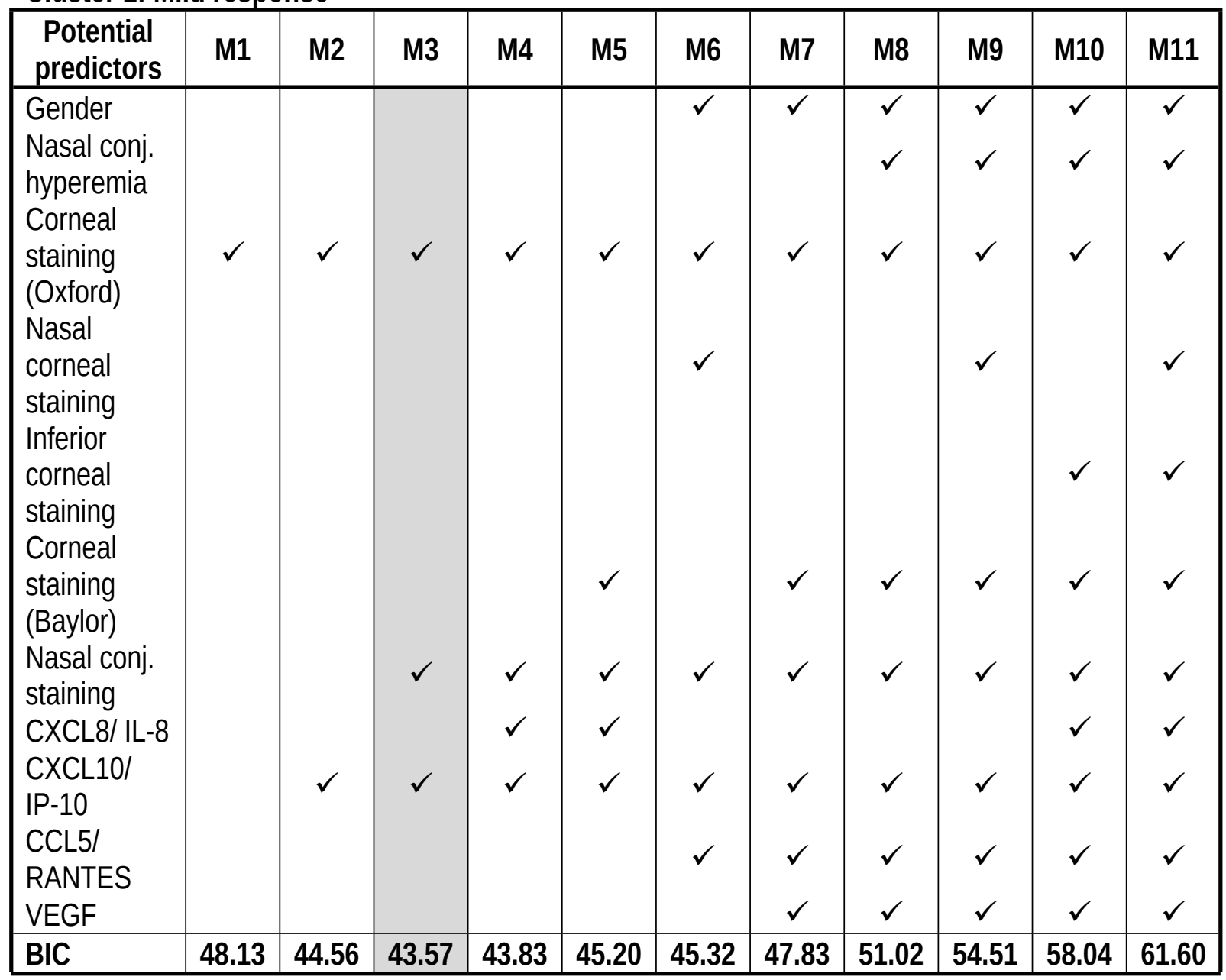

Cluster 2: Corneal epithelial integrity response

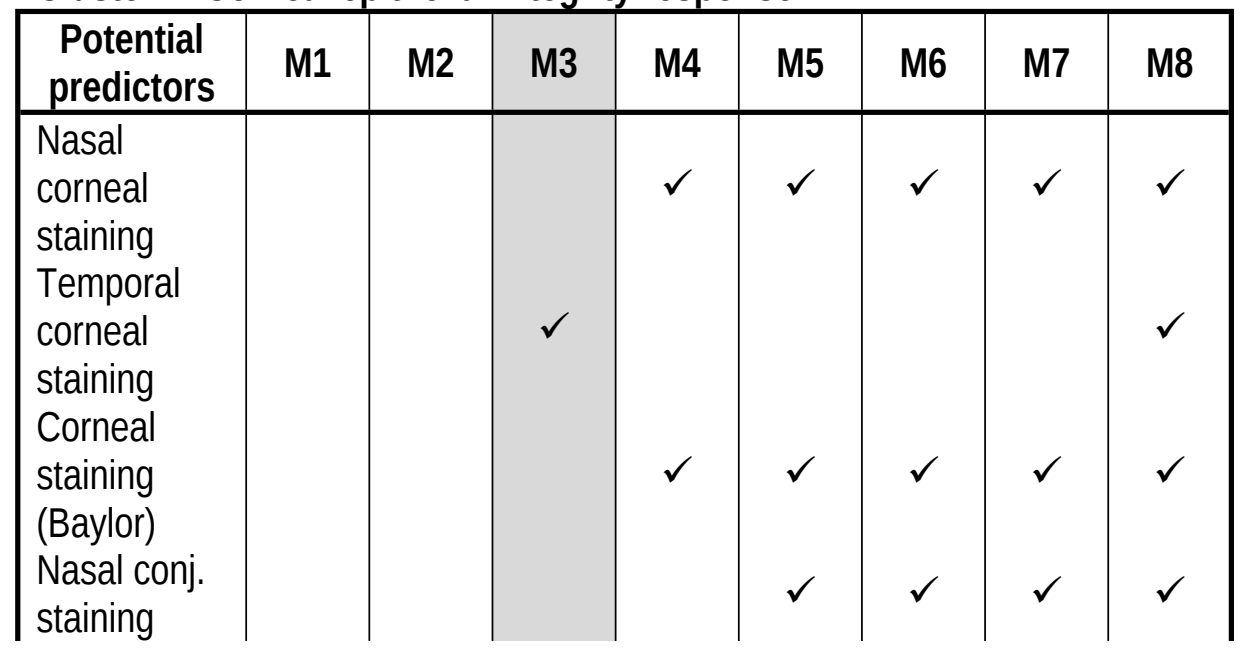




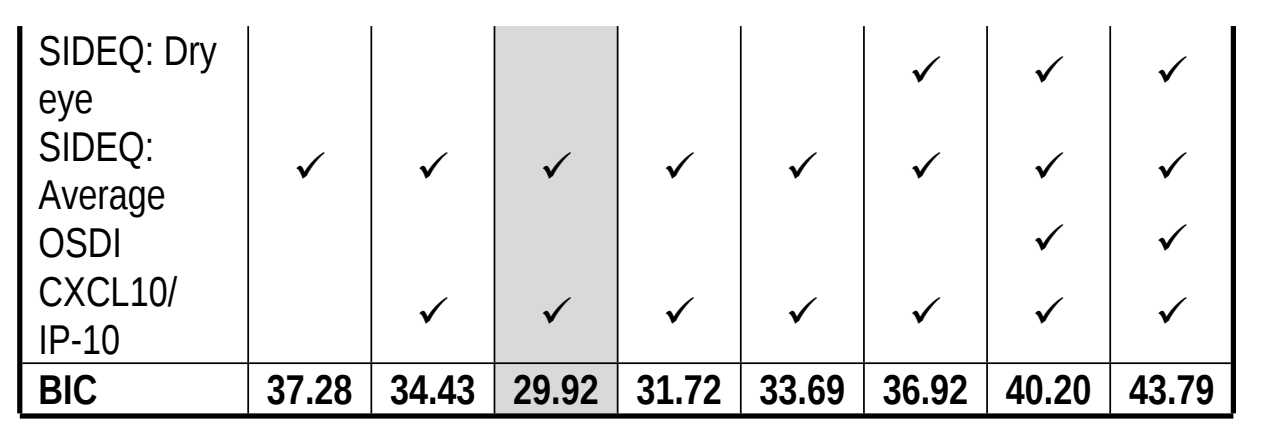

Cluster 3: Tear molecular response

\begin{tabular}{|c|c|c|c|c|c|c|c|c|c|}
\hline $\begin{array}{c}\text { Potential } \\
\text { predictors }\end{array}$ & M1 & M2 & M3 & M4 & M5 & M6 & M7 & M8 & M9 * \\
\hline $\begin{array}{l}\text { Temp. conj. } \\
\text { hyperemia }\end{array}$ & & & & $\checkmark$ & $\checkmark$ & $\checkmark$ & $\checkmark$ & & \\
\hline $\begin{array}{l}\text { Tear } \\
\text { osmolarity } \\
\text { Corneal } \\
\text { staining } \\
\text { (Oxford) }\end{array}$ & & $\checkmark$ & & $\checkmark$ & $\checkmark$ & $\checkmark$ & $\checkmark$ & $\checkmark$ & $\checkmark$ \\
\hline & & & $\checkmark$ & & & & $\checkmark$ & $\checkmark$ & \\
\hline $\begin{array}{l}\text { SIDEQ: Dry } \\
\text { eye }\end{array}$ & & & & & $\checkmark$ & $\checkmark$ & $\checkmark$ & $\checkmark$ & $\checkmark$ \\
\hline $\begin{array}{l}\text { SIDEQ: } \\
\text { Foreign } \\
\text { body }\end{array}$ & & & $\checkmark$ & & & & & & \\
\hline & & & & $\checkmark$ & $\checkmark$ & $\checkmark$ & $\checkmark$ & $\checkmark$ & $\checkmark$ \\
\hline $\begin{array}{l}\text { SIDEQ: } \\
\text { Average }\end{array}$ & & & & $\checkmark$ & $\checkmark$ & $\checkmark$ & $\checkmark$ & $\checkmark$ & $\checkmark$ \\
\hline EGF & & & & & & $\checkmark$ & $\checkmark$ & & $\checkmark$ \\
\hline $\begin{array}{l}\text { CX3CL1/ } \\
\text { Fractalkine }\end{array}$ & & & & & & & & $\checkmark$ & $\checkmark$ \\
\hline $\begin{array}{l}\text { IL-1Ra } \\
\text { CXCL8/ IL-8 }\end{array}$ & & & $\checkmark$ & & & & & & $\checkmark$ \\
\hline $\begin{array}{l}\text { CCL5/ } \\
\text { RANTES } \\
\text { VEGF }\end{array}$ & $\checkmark$ & $\checkmark$ & & & & & & $\checkmark$ & $\checkmark$ \\
\hline$B I C$ & 16.09 & 19.49 & 21.29 & 24.46 & 27.78 & 30.57 & 34.19 & 41.4 & 47.28 \\
\hline
\end{tabular}

Cluster 4: Symptomatic adaptation response

\begin{tabular}{|l|c|}
\hline $\begin{array}{c}\text { Potential } \\
\text { predictors }\end{array}$ & M1 \\
\hline IL-1Ra & $\checkmark$ \\
\hline BIC & 26.92 \\
\hline
\end{tabular}

$\mathrm{BIC}=$ Bayesian Information Criterion; SIDEQ = Single-Item Score Dry Eye Questionnaire; EGF = Epidermal Growth Factor; $\mathrm{CX} 3 \mathrm{CL}=$ Chemokine [C-X3-C motif] ligand; IL-1RA = Interleukin-1 
Receptor Antagonist; $\mathrm{CXCL}=$ Chemokine $[\mathrm{C}-\mathrm{X}-\mathrm{C}$ motif] ligand; IL-8 = Interleukin-8; IP-10 = interferon-gamma- Induced Protein-10; $\mathrm{CCL}=$ Chemokine [C-C motif] ligand; RANTES = Regulated on Activation, Normal T cell Expressed and Secreted; VEGF = Vascular Endothelial Growth Factor.

* In Cluster 3, models based on 10 or more predictors are not valid. Models do not converge. 


\section{APPENDIX B}

Figure B1. Classification trimmed likelihood curves when $\mathrm{k}$ is between 1 and 6 groups and $\alpha$ ranges in $[0,0.2]$ with step size 0.025 trimming proportion. The evaluation of these curves suggests the choice of $k=4$ and $\alpha=0.025$ for applying trimmed $k$-means. There is no clear increase for $k=4$ with respect to the $k=5$ curve over the all range of $\alpha$ values, therefore we choose 4 groups. For $k=4$, parameter $\alpha$ is determined where the initial fast increase of the trimmed classification likelihood curve is stopped.

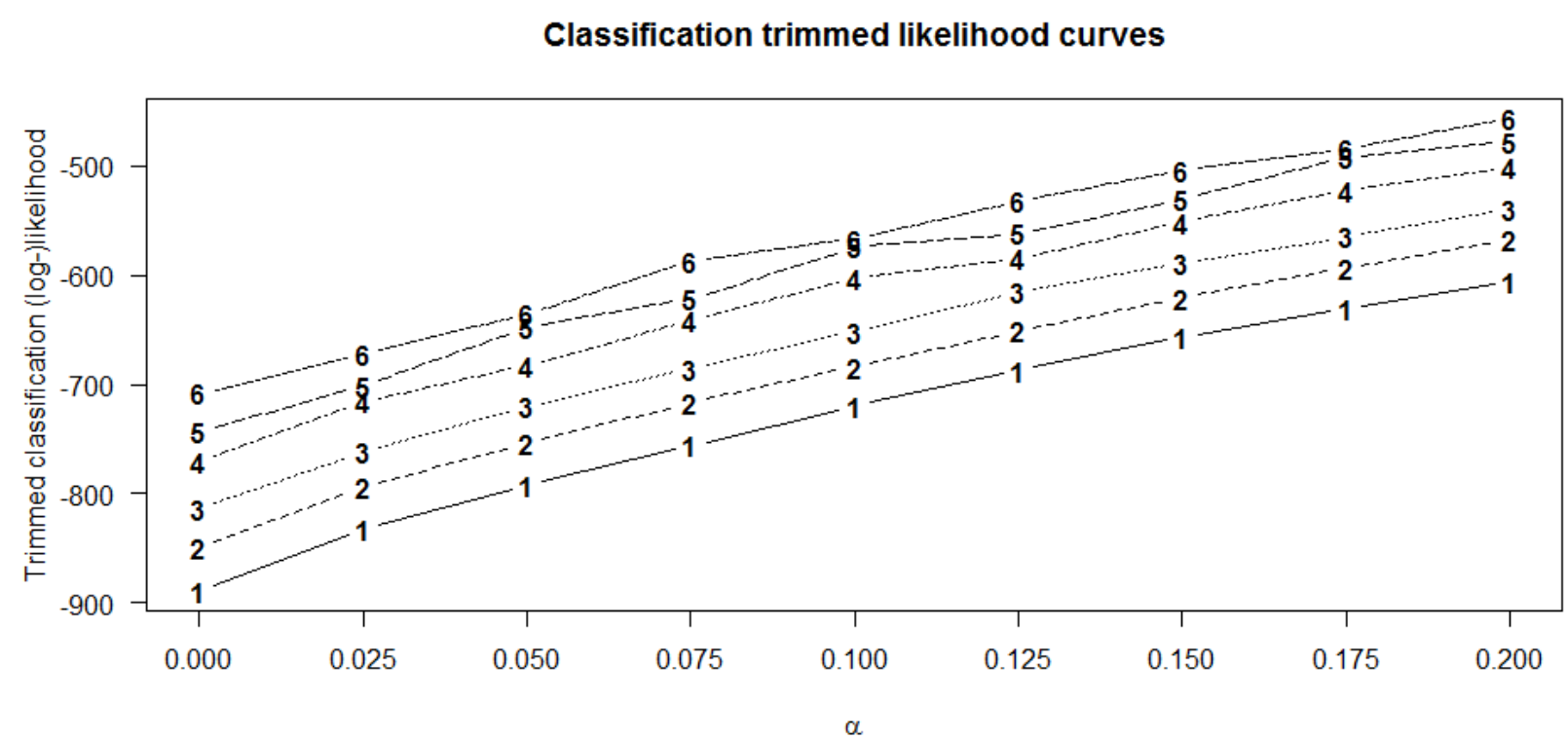




\section{The Ocular Surface}

\section{\&RQMEXKRUKIS[6 WWP HQW}

The journal adheres to the Uniform Requirements set by the International Committee of Medical Journal Editors (http://www.icmje.org/) for authorship. To qualify for authorship of an original research submissions, authors must make substantial contributions to the intellectual content of the paper in each of the four following categories:

1. Substantial contributions to conception and design of the work; or the acquisition, analysis, or interpretation of data for the work; AND

2. Drafting the work or revising it critically for important intellectual content; AND

3. Final approval of the version to be published; AND

4. Agreement to be accountable for all aspects of the work in ensuring that questions related

to the accuracy or integrity of any part of the work are appropriately investigated and resolved.

It is the responsibility of the corresponding author, prior to submitting the manuscript, to confirm that each coauthor meets the requirements for authorship. Please list all authors of the manuscript on the Contributorship Statement form below. The form must be uploaded at the time of original manuscript submission.

By submitting this form, the corresponding author acknowledges that each author has read the statement on authorship responsibility and contribution to authorship. In the table below, please designate the contributions of each author. Any relevant contribution not described in the four columns can be added under "Other contributions." Please note that the list of contributions will publish with the manuscript should it be accepted. Thank you.

\begin{tabular}{|c|c|c|c|c|}
\hline \multicolumn{5}{|l|}{ ? } \\
\hline Author Name & $\begin{array}{c}\text { Research design } \\
\square\end{array}$ & $\begin{array}{c}\text { Data acquisition } \\
\text { and/or research } \\
\text { execution } \\
\end{array}$ & $\begin{array}{l}\text { Data analysis } \\
\text { and/or } \\
\text { interpretation } \\
\end{array}$ & $\begin{array}{c}\text { Manuscript } \\
\text { preparation } \\
\end{array}$ \\
\hline Itzliar]Fernández & $\square \square$ & $\square \square$ & $\square \square$ & $\square \square$ \\
\hline Albeqto López-M & $\square \square$ & $\square \square$ & $\square \square$ & $\square \square$ \\
\hline Analia Enríquez & $\square \square$ & $\square \square$ & $\square \square$ & $\square \square$ \\
\hline Marisa Tesón & $\square \square$ & $\square \square$ & $\square \square$ & $\square \square$ \\
\hline Michaele Stern & $\square \square$ & $\square \square$ & $\square \square$ & $\square \square$ \\
\hline Mąria JIGonzále & $\square \square$ & $\square \square$ & $\square \square$ & $\square \square$ \\
\hline Marogarita Calons & $\square^{\square}$ & $\square \square$ & $\square \square$ & $\square \square$ \\
\hline 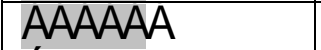 & $\square \square$ & $\square \square$ & $\square \square$ & $\square \square$ \\
\hline
\end{tabular}

Other contributions: 